VERsion OCTOBER 16, 2018

Preprint typeset using $\mathrm{L}^{\mathrm{A}} \mathrm{T} \mathrm{E}$. style emulateapj v. 05/12/14

\title{
THERMONUCLEAR SUPERNOVAE: PROBING MAGNETIC FIELDS BY POSITRONS AND LATE-TIME IR
} LINE PROFILES

\author{
R. Penney ${ }^{1,2}$, P. Hoeflich ${ }^{1}$ \\ Version October 16, 2018
}

\begin{abstract}
We show the importance of $\gamma$ and positron transport for the formation of late-time spectra in Type Ia Supernovae (SNe Ia). The goal is to study the imprint of magnetic fields $(B)$ on late-time IR line profiles, particularly the [FeII] feature at $1.644 \mu \mathrm{m}$ which becomes prominent two to three months after the explosion. As a benchmark, we use the explosion of a Chandrasekhar mass $\left(M_{C h}\right)$ White Dwarf (WD) and, specifically, a delayed detonation model which can reproduce the light curves and spectra for a Branch normal SNe Ia. We assume WDs with initial magnetic surface fields between 1 and $10^{9} \mathrm{G}$. We discuss large-scale dipole and small-scale magnetic fields. We show that positron transport effects must be taken into account for the interpretation of emission features starting at about 1 to 2 years after maximum light, depending on the size of $B$. The [FeII] line profile and its evolution with time can be understood in terms of the overall energy input by radioactive decay and the transition from a $\gamma$-ray to a positron dominated regime. We find that the [Fe II] line at $1.644 \mu \mathrm{m}$ can be used to analyze the overall chemical and density structure of the exploding WD up to day 200 without considering $B$. At later times, positron transport and magnetic field effects become important. After about day 300 , the line profile allows one to probe the size of the $B$ field. The profile becomes sensitive to the morphology of $B$ at about day 500. In the presence of a large-scale dipole field, a broad line is produced in $M_{C h}$ mass explosions which may appear flat-topped or rounded depending on the inclination at which the supernova is observed. Small or no directional dependence of the spectra is found for small-scale $B$. We note that narrow line profiles require central ${ }^{56} \mathrm{Ni}$ as shown in our previous studies. Persistent broad-line, flat-topped profiles require high density burning which is the signature of a WD close to $M_{C h}$. Good time coverage is required to separate the effects of optical depth, the size and morphology of $B$, and the aspect angle of the observer. The spectra require a resolution of about $500 \mathrm{~km} / \mathrm{sec}$ and a signal to noise ratio of about $20 \%$. Two other strong NIR spectral features at about 1.5 and $1.8 \mu \mathrm{m}$ are used to demonstrate the importance of line blending which may invalidate a kinematic interpretation of emission lines. Flat-topped line profiles between 300 and 400 days have been observed and reported in literature. They lend support for $M_{C h}$ mass explosions in at least some cases, and require magnetic fields equal to or in excess of $10^{6} \mathrm{G}$. We briefly discuss the effects of the size and morphology of B on light curves, and limitations. We argue that line profiles are a more direct measurement of $B$ than LCs because they measure both the distribution of ${ }^{56} \mathrm{Ni}$ and the redistribution of the energy input by positrons rather than the total energy input. Finally, we discuss possible mechanisms for the formation of high B-fields, and the limitations of our analysis.
\end{abstract}

Subject headings: Supernovae: general — positron transport — IR spectra

\section{INTRODUCTION}

Type Ia supernovae (SNe Ia) are invaluable fro probing the large-scale structure of the Universe and the origin of the elements. They also provide important laboratories for the study of the physics of flames, instabilities, radiation transport, non-equilibrium systems, and nuclear and high energy physics. The consensus picture is that SNe Ia result from a degenerate $\mathrm{C} / \mathrm{O}$ white dwarf undergoing a thermonuclear runaway (Hovle \& Fowler 1960), and that these in turn originate from close binary stellar systems. Potential progenitor systems may either consist of two WDs, a so called double degenerate system (DD), and/or a single WD and a main sequence, Helium or Red Giant star, called a single degenerate system (SD). Candidate progenitor systems have been observed for both the SD and DD cases (Greiner et al. 1991; van den Heuvel et al. 1992; Rappaport et al. 1994; Kahabka \& van den Heuvel 1997; Ruiz-Lapuente et al. 2004; González Hernández et al. 2009; Kerzendorf et al. 2009; Schaefer \& Pagnotta| 2012; Edwards et al. 2012). For recent overviews, see proceedings of the IAU 281 Symposium edited by DiStephano \& Orio (2012).

Within this general picture for progenitors, two classes of explosion scenarios have been discussed, which are distinguished by the triggering mechanism of the thermonuclear explosion. The first is the dynamical merging of two $\mathrm{C} / \mathrm{O}$ white dwarfs in a binary system. In this scenario, the thermonuclear explosion is triggered by the heat of the merging process. However, it is unclear whether the dynamical merging process leads to a SN Ia, an "Accretion Induced Collapse", or a WD with high a magnetic field. Another problem is that there seem to be too few potential progenitor systems (Webbink 1984; Iben \& Tutukov 1984; Benz et al. 1990; Rasio \& Shapiro 1994; Hoeflich \& Khokhlov 1996;

\footnotetext{
${ }^{1}$ Florida State University, Department of Physics, Tallahassee, FL 32305, USA, phoeflich77@gmail.com
}

2 Clemson University, Tallahassee, FL 32305, USA, rpenney@g.clemson.edu 
Segretain et al. 1997; Yoon et al. 2007; Wang et al. 2009b, a; Pakmor et al. 2011; Lorén-Aguilar et al. 2009; Isern et al. 2011).

The second scenario involves the explosion of a C/O WD with a mass close to the Chandrasekhar limit $\left(M_{C h}\right)$. The explosion in this scenario is triggered by compressional heating near the WD center. Because the compressional heat released increases rapidly as the star nears $M_{C h}$, the exploding stars are in a very narrow mass range (Hoeflich \& Khokhlov 1996). The donor star may be either a red giant or main sequence star of less than 7-8 solar masses or a helium star in an SD-system (Whelan \& Iben 1973), or the accreted material may originate from a tidally disrupted WD in a DD-system (Whelan \& Iben 1973; Piersanti et al. 2003).

A comprehensive discussion of the progentors and explosion mechanisms is beyond the scope of this paper. For reviews and different views, see (Branch \& Miller 1993; Pakmor et al. 2011; Calder et al. 2013; Guidry \& Messer 2013; Hoeflich et al. 2013). Theoretical work and observational constraints from the spectra and light curves favor the single degenerate scenario for the majority of cases, with some contribution from the double degenerate scenario (Hoeflich \& Khokhlov 1996; Saio \& Nomoto 1998; Wooslev \& Weaver 1986; Mochkovitch \& Livio 1990; Saio \& Nomoto 1985; Quimbv et al. 2006; Shen et al. 2011; Hoeflich et al. 2013). Within $M_{C h}$ mass explosions, delayed-detonation models (Khokhlov 1991; Wooslev \& Weaver 1994; Yamaoka et al. 1992; Gamezo et al. 2003; Gamezo et al. 2005; (Poludnenko et al.|2011). Models possessing a transition from a deflagration to a detonation front (DDT), have been found to reproduce the optical and infrared light curves and spectra of individual "typical" SNe Ia reasonably well, including the time evolution (Hoflich 1995; Hoeflich \& Khokhlov 1996; Fisher et al. 1997; Nugent 1997; Wheeler et al. 1998; Lentz et al. 2001; Marion et al. 2009; Maund et al. 2010; Sim et al. 2013; Dessart et al. 2014) and statistical properties, such as the small spread in the brightness-decline relation (Hoeflich et al. 1996; Nugent et al. 1997; Hoeflich et al. 2002; Maeda et al. 2003; Kasen et al. 2009; Baron et al. 2012). The overall spherical structure in SN Ia remnants and, in particular, the layered chemical structure observed in S-Andromeda are clear evidence of a detonation phase (Fesen et al. 2007). Though small, deviations from sphericity are to be expected due to rotation of the WD, off-center DDT and turbulence in the deflagration phase. These have been shown to be present in studies of spectropolarization, IR line profiles and supernovae remnants (Howell et al. 2001; Hoeflich et al. 2004; Motohara et al. 2006; Hoeflich et al. 2006; Gerardy et al. 2007; Fesen et al. 2007; Maund et al. 2010; Fesen et al. 2007; Maeda et al. 2011). Recently, correlations between line profiles and light curves (Maeda et al. 2011) and line profiles and polarization (Maund et al. 2010) promise further progress towards a more complete picture of SNe Ia.

We regard four properties of SNe Ia as the primary evidence in favor of $M_{C h}$ explosions: A tight relation of the absolute brightness with the rate of the decline in brightness; low continuum polarization (Wang et al. 1997; Howell et al. 2001; Patat et al. 2012); evidence for nuclear burning at densities $>10^{9} \mathrm{~g} / \mathrm{cm}^{3}$ from late-time IR spectra (Hoeflich et al. 2004; Motohara et al. 2006; Maeda et al. 2011) and narrow Ni lines at late times as observed in SN 2003hv (Gerardy et al. 2007). In SN 2003hv, late-time MIR spectra at about 130 days have been obtained with the Spitzer Space Telescope. They show flat-top profiles consistent with the NIR profile and, thus, support the kinematic interpretation. Whereas the forbidden Co lines are broadly consistent with the expected distribution of ${ }^{56} \mathrm{Ni}$, late time $N i$ lines are found to be narrow. Since ${ }^{56} N i$ has a decay time of about 6.3 days, after several months only stable $N i$ isotopes are left, and they exist only in the central region (Gerardy et al. 2007; Stritzinger et al. 2014). Thus, at least some supernovae originate from $M_{C h}$ mass explosion.

Despite the success of $M_{C h}$ mass explosions, whether they originate from SD or DD systems, there are serious problems within this picture related to the mixing by Rayleigh-Taylor instabilities (R-T) inherent in current multi-dimensional simulations of deflagration fronts (Khokhlov 1995; Niemeyer \& Hillebrandt 1995; Livne 1999; Reinecke et al. 1999; Gamezo et al. 2003; Plewa 2007; Röpke et al. 2006). During the deflagration phase, the unburned material is heated by the diffusion of energy from the burned ashes to the unburned fuel. R-T instabilities increase the surface area of the burning front and, thus, control the rate of burning while mixing the burning products of different layers. Although a layered chemical structure is partially restored during the detonation phase (Gamezo et al. 2005; Röpke et al. 2012), all current 3D models for the explosions predict R-T mixing of iron-group plumes into the outer layers and mixing of the inner layers, both of which are at odds with observations.

To understand the inconsistencies, it is necessary to both probe the distribution of radioactive material in SNe Ia and to look for new physical effects not considered in current 3D simulations for deflagration fronts. Late-time line profiles of [Fe II] and [Co III] probe the distribution (Hoeflich et al. 2004; Motohara et al. 2006; Maeda et al. 2011) and provided evidence for high magnetic fields in excess of $3000 \mathrm{G}$ (Hoeflich et al.|2004; Sadler 2012), a piece of physics which may influence the runaway and burning instabilities (see below). Moreover, advances in observational techniques have allowed measurement of spectra at late times and thus are providing an increasingly comprehensive set of SNe Ia data (Phillips 2012; Stritzinger et al. 2014).

The distribution of elements and the strength and structure of $B$ may be a key to a better understanding of SNe Ia. However, depending on the phase, a direct translation of the Doppler shifts of a spectral feature is limited by optical depth effects, line blending, and a mix of geometrical asymmetries in the distribution. Moreover, a quantification of the $B$ fields requires detailed gamma and positron transport. Past studies of line profiles were based on the assumption of local energy deposition of positrons.

The goal of this work is to present a systematic study of the effect of $B$ fields on the NIR line features. We use a spherical density and chemical distributions and base our study on a DDT model as reference. We employ detailed Monte Carlo schemes for the transport for photons, gamma-rays and positrons. In section 2, we will present a new positron transport scheme, and briefly summarize the other tools employed for the simulations. In the following 
sections, the results are presented and discussed. We will present a systematic study of the evolution of NIR profiles of [Fe II] and [Co III] in the 1.6 to $1.7 \mu \mathrm{m}$ region. We show the importance of time-sequences to untangle the various effects.

\section{METHODS}

Our analysis of the line profiles is based on the delayed detonation model 5p0z00.25, a 'Branch-normal' Type Ia which is part of the series published by Hoeflich et al. (2002). As in our previous analysis of the NIR [Fe II] line (Hoeflich et al. 2004), the 3D-transport is calculated using a Monte Carlo scheme implemented in HYDRA, which includes detailed $\gamma$-ray transport. Forbidden lines are included from databases published by Kurucz (1993, 2002); Nussbaumer \& Storey (1988b a ); Liu et al. (1997a); Bowers et al. (1997). The new addition here involves the energy deposition by positrons and the extension from $\gamma$ to X-rays. These new modules will be described in the following.

\subsection{Positron Transport Scheme}

Several previous investigators have studied the transport of energy by high energy photons through supernova envelopes (Ambwani \& Sutherland 1988a; Burrows \& The 1990; Chan \& Lingenfelter 1991; Hoeflich et al. 1994; Hoeflich 2002). As part of his PhD thesis, new bound-free and bound-bound transitions have been implemented which allows us to calculate X-ray spectra (Pennev 2011). This transport module has been further extended to incorporate positron transport in the presence of magnetic fields.

Several authors have addressed the transport of positrons (Bussard et al. 1979; Brown \& Leventhal 1987). Early studies by Colgate et al. (1980) and Chan \& Lingenfelter (1993) used one-dimensional approximations and assumed the magnetic field was either chaotically twisted at a scale small enough to trap the positrons in place or radially combed by the expansion, restricting the positrons to move radially. They calculated the energy deposition by positrons at late times and demonstrated the extent to which positron escape would lead to light-curves (LCs) declining faster than the rates of the radioactive decay driving them. Milne et al. (1999) presented calculations using cross-sections and a Monte-Carlo technique more similar to ours, but continued to use either a radial magnetic field or ones which are chaotic and sufficiently large to trap all positrons. As part of the thesis of Pennev (2011), light curve results have been compared to those published Milne et al. (2004). As discussed below, we found a generally good agreement, but with a slightly reduced positron escape. In this paper, we will make use of line profiles to measure positron transport effects and their dependency on $B$. Profiles can be expected to more sensitive and 'cleaner' than LCs because they measure directly the change in the redistribution function of the energy deposition from radioactive decay. In contrast, LCs rely on one quantity, the total energy deposition in the envelope that powers the bolometric LC. The reconstruction of the bolometric LC depends in turn on the photon redistribution in frequency space and, after a few years, other energy sources may become important such as interaction between the SN and the interstellar material (ISM).

\subsection{Positron Creation and Cross Sections}

The primary source of positrons is the $\beta^{+}$channel of the ${ }^{56} \mathrm{Co} \rightarrow{ }^{56} \mathrm{Fe} e^{*}$ which accounts for about $18 \%$ of all ${ }^{56} \mathrm{Co}$ decays. About $1.4 \mathrm{MeV}$ of the total excitation energy of ${ }^{56} \mathrm{Fe} e^{*}$ is available to be split between neutrinos and positrons , with the resulting positron energy spectrum (Nadyozhin 1994) given by

$$
N(E)=C p^{2}\left(E_{o}-E\right)^{2}\left(2 \pi \eta(1-\exp (-2 \pi \eta))^{-1}\right)
$$

where $C$ is a scale factor and $\eta$ is the charge of the nucleus times $\hbar$ over the velocity of the electron. The mean energy of the spectrum is .632 MeV.

A small number of relatively high energy positrons are also contributed by pair-production from the high-energy gamma-ray lines of ${ }^{56} \mathrm{Co}$ decay. As most $\gamma \mathrm{s}$ escape without interaction at late times, these contribute only a few percent to the total positron production by 300 days, but their production and transport is included. The cross-section for this channel of positron production has been discussed by Ambwani \& Sutherland (1988b).

For the Monte Carlo transport, we take into account three dominant processes of interaction: scattering off atomic electrons as the positron moves through the media, the similar excitation of free electrons in a plasma, and direct annihilation with electrons.

The annihilation cross-section is given by eq. 2 (Lang 1999):

$$
\sigma=\frac{\pi r_{o}}{\gamma+1}\left[\frac{\gamma^{2}+4 \gamma+1}{\gamma^{2}-1} \ln \left(\gamma+\sqrt{\gamma^{2}-1}\right)-\frac{\gamma+3}{\sqrt{\gamma^{2}-1}}\right]
$$

with $r_{o}=e^{2} / m c^{2}$ and $\gamma$ is the Lorentz factor. In undergoing this interaction, the positron is destroyed, its kinetic energy is deposited into the surrounding material and its rest-mass energy is released as gamma-rays.

Following Chan \& Lingenfelter (1993) and Gould (1971), the energy loss by interaction with charged particles is given by

$$
\frac{d E}{d x}=\frac{-4 \pi r_{o}^{2} m_{e} c^{2} q}{A M_{n} \beta^{2}}\left(q \ln \left(\frac{\sqrt{\gamma-1} \gamma \beta m_{e} c^{2}}{b_{\max }}\right) \Pi(\gamma)\right.
$$

$\Pi(\gamma)$ is a relativistic correction given by

$$
\Pi(\gamma)=\frac{\beta^{2}}{12}\left[\frac{23}{2}+\frac{7}{\gamma+1}+\frac{5}{(\gamma+1)^{2}}+\frac{2}{(\gamma+1)^{3}}\right]
$$




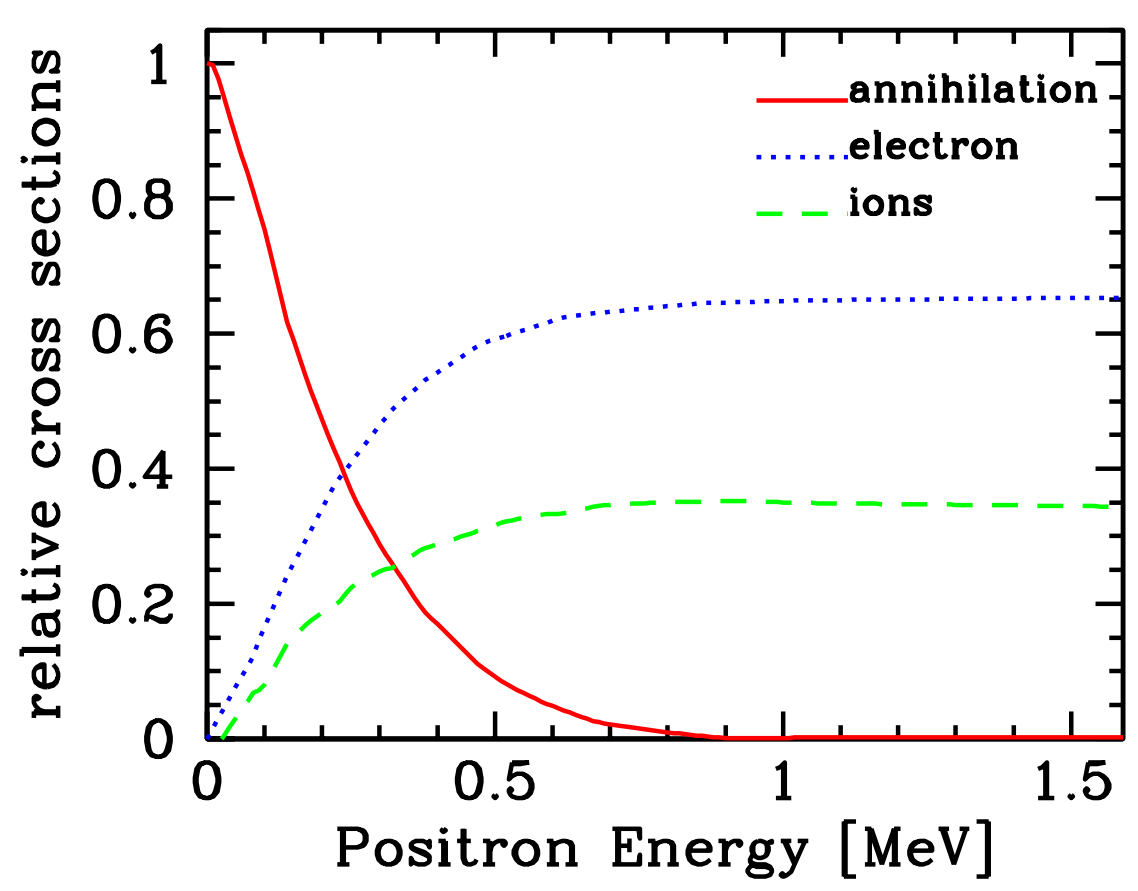

FIG. 1. - The strength of the electron, plasma, and annihilation cross sections for a single ionized plasma. Annihilation dominates at low energies after the positrons lose most of their energy by electron and plasma interaction. Electrons dominate plasma interactions for low ionization levels but ions will dominate in highly ionized plasmas.

where $q$ is the relative charge of the particles in the media. For atomic electron interactions, $q$ is the atomic number $Z$ for the atom. For plasma scattering, $q$ is the ionization fraction. $b_{\max }$ is the maximum impact parameter, equivalent to the maximum amount of energy the positron can loose in one interaction. For atomic electron scattering, it is the the ionization potential. Since the dependence on this parameter is weak, we simplify by using an average ionization potential for the medium. For the case of interactions with electrons in the plasma, the maximum impact parameter is $\hbar \omega$ because the impact with a free electron sets up a disturbance in the plasma which has an energy proportional to the frequency. Note that the ionization stage depends on time and requires ionization models which, in turn, will depend on the energy deposition by positrons. In this study, we assume single ionization. Other sources of continuous energy loss, those of Bremsstrahlung and synchrotron radiation, were considered but found to be minor contributions and, for computational efficiency, have been omitted in this study.

The relative strengths of the three types of cross-sections are shown in Fig. 1] Annihilation is a very small part of the cross-section through-out most of the energy range of the positron spectrum. As the positrons scatter and continuously loose energy, though, they will either fall into the range where direct annihilation dominates and they be destroyed, or they will slow down until their energies become similar to the binding energy of atomic electrons, form positronium, and be annihilated.

Between $1 \mathrm{KeV}$ and the binding energy of nearby electrons, the positron can undergo charge exchange, stripping an electron off nearby atoms to form positronium, which has a negligible lifetime before undergoing either $e^{+} e^{-} \rightarrow 2 \gamma$ or $e^{+} e^{-} \rightarrow 3 \gamma$ for para- and ortho-positronium, respectively. We assume that the para- and ortho-state of positronium are in statistical equilibrium for the creation of $\gamma$ photons. The cross-section of this process for elements other then $H$ is not well known, but is generally proportional to the Bohr-radius, and thus much larger then the direct annihilation cross-section.

This uncertainty in the cross-section for annihilation at low energies will hardly influence the location of the energy deposition because the energy loss is large (see eq. 3). Even at day 1000 after the explosion, the range of a 1 KeV positron is less than $0.1 \%$ of the radius of the envelope. Thus, we assume that once a positron has fallen below $1 \mathrm{KeV}$, it deposits its kinetic energy locally and annihilates via positronium formation. Chan \& Lingenfelter (1993) found that some small fraction of thermalized positrons $(\approx .1 \%$ in models similar to ours) will avoid annihilation , surviving as "slow" positrons as the envelope density becomes low enough for the lifetime before positronium formation to become very long. However, as these will have already deposited their kinetic energy in the ejecta, and their mass-energy would not contribute whether they annihilate or not. At late times, $\gamma \mathrm{s}$ from annihilation are almost guaranteed to escape the envelope without interaction.

\subsection{Positron Transport:}

The transport is solved via a Monte Carlo method very similar to the photon transport (Hoeflich et al. 1994; Hoeflich 2002). A background model is discretized and the radiation transport is performed in individual computational cells. 
TABLE 1

MAXIMUM LAMOR RADIUS AS A FRACTION OF THE RADIUS OF THE SUPERNOVA ENVELOPE AT VARIOUS TIMES AND INITIAL SURFACE FIELD STRENGTHS. HERE, THE RADIUS IS DEFINED AS THE LAYER EXPANDING WITH A VELOCITY OF $40.000 \mathrm{KM} /$ SEC. TYPICALLY, TURBULENT VELOCITIES IN THE PROGENITOR AND DURING THE DEFLAGRATION PHASE OF BURNING ARE BETWEEN $\approx 200 \mathrm{TO} \approx 1000 \mathrm{~km} / \mathrm{sec}$ (HoEflich \& Stein 2002; GaMezo ET AL. 2005), OR $5 \times 10^{-3}$ AND $2.5 \times 10^{-2}$ IN Units of the FRACtions Given.

\begin{tabular}{|c|c|c|c|c|c|}
\hline field/time & $20 \mathrm{~d}$ & $60 \mathrm{~d}$ & $100 \mathrm{~d}$ & $300 \mathrm{~d}$ & $500 \mathrm{~d}$ \\
\hline $10^{3} \mathrm{G}$ & .38 & 1.15 & 1.9 & 5.7 & 9.6 \\
\hline $10^{4} \mathrm{G}$ & .03 & .11 & .19 & .57 & .96 \\
\hline $10^{6} \mathrm{G}$ & .003 & .001 & .001 & .005 & .009 \\
\hline $10^{9} \mathrm{G}$ & $3 E-6$ & $1.1 E-6$ & $1.9 E-6$ & $5 E-6$ & $9 E-6$ \\
\hline
\end{tabular}

Upon interaction, the process is chosen randomly, weighted by the size of the individual cross-sections.

The motion of positrons depends on the presence, size and morphology of the magnetic field $B$ imposing a Lorentz force on the positron. The positron gyros around the magnetic field line. The scale imposed by $B$ is the Lamour radius

$$
r_{L}=\frac{p_{\perp}}{q B} \quad\left(p_{\perp} \text { the momentum perpendicular to the field. }\right)
$$

The significance of the gyro-motion depends on the size of $r_{L}$ relative to the scale of variations of the explosion model $r_{M}$, such as the density scale height or the scale of the $B$ field changes.

For computational efficiency, we distinguish three different implementations: 1) $\left.r_{L}>>r_{M}, 2\right) r_{L}<<r_{M}$, and 3) the case in between.

In case 3 , the path of the positron must be explicitly integrated by breaking the path-length into segments sufficiently small so that the B-field changes little along individual segments. A positron propagates along a circular arc on that path (the radius determined by eq. 5) until it is absorbed, scatters, or escapes the envelope.

Case 1 and 2 avoid the need to reconstruct the path for individual positrons and, thus, they allow for a higher computational efficiency by a factor of more than $\approx 10$.

For case 1, small magnetic fields, the path of the positron is nearly straight and we integrate along linear rays until the positron is absorbed, scattered or escapes.

For large magnetic fields, $r_{L}$ is small compared to the structure of the explosion model. The positron can be assumed to follow the field line on a spiral trajectory mean-free path. The general motion of a positron is along the field line with its path length increased by a factor of $1 / \sin \left(\theta_{p}\right)$, where $\theta_{p}$ is the pitch angle the positrons momentum vector makes with the field line. Effects of gradients in $B$ are implemented following Chen (2003). We divide these into two forces, one parallel to the field lines, and one perpendicular. The former causes the "magnetic mirror" effect. Because the first adiabatic invariant is constant, a change in the B field strength along the direction of travel causes a change in the pitch angle. In the code, we average the derivative of the B field over the zone in the direction of the field lines, and use this to calculate an average gyro-radius for that zone. The result is that positron diffusion is discouraged in the direction of increasing magnetic field and encouraged in the opposite direction, as an increasing pitch angle will increase the pathlength. The effect of the perpendicular component of the $B$ gradient causes the center of the gyro-radius off the field line due to gradients perpendicular to the $B$ field. We add this velocity to the motion of the particle in each zone using the following equation

$$
v=\frac{1}{2} v_{\perp} r_{L} \frac{B \times \nabla B}{B^{2}}
$$

For illustration, table 1 shows the evolution of $r_{L}$ for a typical SNIa model as a function of time. In practice, we use cases 3 or 1 if $r_{L}$ is smaller than the resolution of the background model or larger than twice the model grid, respectively. Cases 1 and 3 have been tested against the general case 2 . With time and lower magnetic fields, the range of the positron transport increases. For $B$ fields larger than $10^{9} G$, positrons follow the field lines. For $B$ fields less than $10^{3} G$ and about one year, the bending of the path of the positrons remains small and they travel along rays. We note that turbulence will produce structures of about 1 to $5 \%$ of the envelope structure. Thus, the energy deposition by positrons requires full transport of positrons for all $B$ fields less than $B \approx 10^{6} G$ when the transport effects are important.

\section{THE REFERENCE MODEL}

This study uses a spherical, delayed detonation model as a reference model. This model has been successful in reproducing the optical and IR light curves and spectra of a Branch normal SNe Ia, 5p0z22.25 (Hoeflich et al. 2002). The $\mathrm{C} / \mathrm{O}$ white dwarf originates from a progenitor with a main sequence mass of $5 M_{\odot}$ and solar metallicity. At the time of the explosion, the central density of the WD was $2 \times 10^{9} \mathrm{~g} / \mathrm{cm}^{3}$, and the transition from deflagration to detonation was triggered at a density of $25 \times 10^{6} \mathrm{~g} / \mathrm{cm}^{3}$. About $0.6 M_{\odot}$ of ${ }^{56} \mathrm{Ni}$ is produced. The density, velocity and chemical structure is given in Fig 2. As typical for $M_{C h}$ mass explosions, the high density burning in the central region produced stable isotopes of iron-group elements rather than radioactive ${ }^{56} \mathrm{Ni}$. Spherical models suppress mixing by Rayleigh-Taylor instabilities and, as a consequence, we produce a central 'hole' in the ${ }^{56} \mathrm{Ni}$ distribution of about $3000 \mathrm{~km} / \mathrm{sec}$. The spherical explosion model and evolution has been calculated using 912 radial zones in the comoving frame. Positron transport is inherently 3-dimensional in presence of $B$ fields. For the $\gamma$ and positron and NIR transport, 

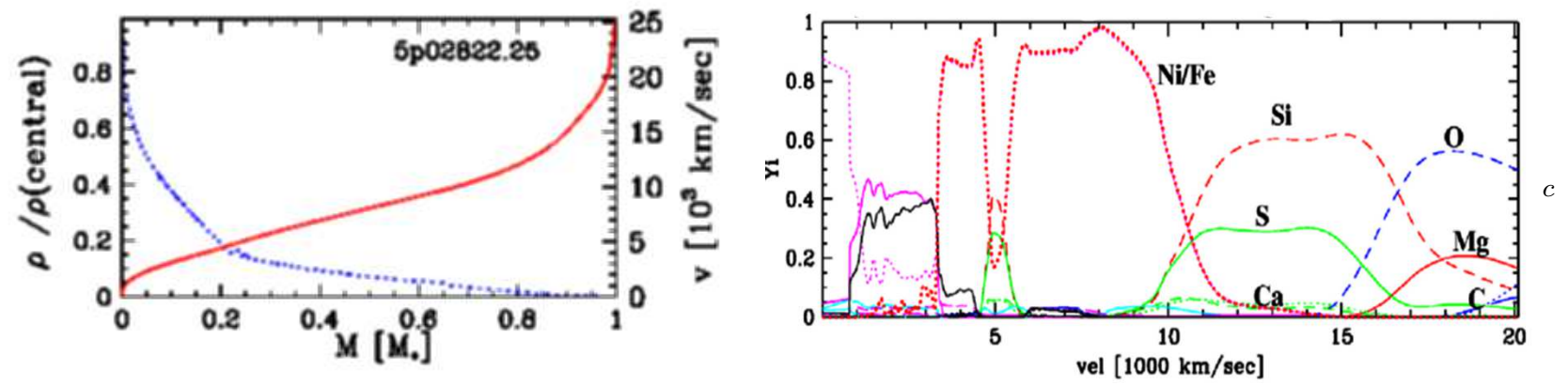

FIG. 2.- Structure of a spherical delayed detonation model which can reproduce LCs and spectra of Branch-normal SNe Ia (Hoeflich et al. 2002). Density (blue, dotted) and velocity (red, solid) as a function of the mass are given on the left. Abundances of the most abundant stable isotopes as a function of the expansion velocity are given on the right. In the center, the abundances correspond to ${ }^{54} \mathrm{Fe},{ }^{58} \mathrm{Ni}$ and Co. We note that current 3D calculations predict similar density structures but with strong chemical mixing.

the model has been remapped into the observers frame using an Euclidian grid with 330 radial zones with a resolution of about $50 \mathrm{~km} / \mathrm{sec}$ up to the Si-rich layers. For $\theta$ and $\phi$, we use 70 zones with equidistant spacing in $\sin \theta$ and $\phi$. The energy deposition has been stored on a Cartesian grid of $501^{3}$ with a matching resolution of $100 \mathrm{~km} / \mathrm{sec}$. The emitted photons have been detected in 21 counters each both in $\theta$ and $\phi$, and about 2500 counters in frequency/energy. The averaged positron spectrum is detected in 100 counters. A typical run uses a package of $1-510^{10}$ photons and positrons.

To study the effects of $B$, we imprint various fields onto the model described above. We assume that the initial field prior to the explosion of the WD can be described as a dipole with a surface averaged field of $B$. For the rotation, we assume that the WD is a rigid rotator with angular velocity $w$, and a constant magnetization $m$. Taking the field due to a thin spherical shell and summing to the radius of the dwarf $R_{o}$ (taking $\mathrm{R}$ to be the radius of the White Dwarf at $90 \%$ of the Chandrasekhar Mass, $1600 \mathrm{~km}$ ), the structure of the field is given by

$$
\overrightarrow{B_{o}}(r, \theta)=\omega m\left[\left(1-\frac{3 r^{2}}{5 R_{o}^{2}}\right) \cos (\theta) \hat{r}-\left(1-\frac{6 r^{2}}{5 R_{o}^{2}} \sin (\theta)\right) \hat{\theta}\right]
$$

$B$ field strengths are considered between 1 and $10^{9} \mathrm{G}$. With the surface field as boundary condition, we use equation 7 to calculate the magnetic field throughout the progenitor. Note that the maximal range of the field strength is larger by about a factor of 4 close to the center of the initial WD. We can assume that the magnetic field is frozen into the matter during the explosion. The final structure of the field is very similar because of the similarity of the initial structure and the homologous expansion of the WD. After the initial acceleration phase, a few minutes into the expansion, the time evolution of the field at every point decreases as the square of the radius and thus, with time because of conservation of the magnetic flux. During the homologous expansion field strength at $r$ is given by $B_{o}\left(\frac{r_{o}}{r}\right)^{2}$.

\section{RESULTS}

In this section, we will discuss the use of IR lines as tool for studying the underlying chemical structure and the signature of the strength and structure of the magnetic fields at late times. The use of LCs is also considered briefly, and compared to that of IR lines. Both light curves and spectral line profiles allow the probing of magnetic fields. The advantage of broad-band light curves are that they provide high photon statistics. This allows one to obtain accurate LCs until very late-times and, in principle, for a large number of objects. However, late time LCs contain limited information because they mostly depend on one quantity only: the combined escape probability of $\gamma-$ rays and positrons. Spectra contain information about the escape probability and the time-dependent redistribution functions but limiting photon statistics put high demands on the observations and limits their use to 'local' supernovae. Both the resulting LCs and spectra can be understood in terms of the energy deposition.

\subsection{Energy Deposition by $X$ - and $\gamma$-Rays and Positrons}

Radioactive decay of ${ }^{56} \mathrm{Ni} \rightarrow{ }^{56} \mathrm{Co} \rightarrow{ }^{56} \mathrm{Fe}$ is the dominant energy source which powers the light curves and spectra of SNe Ia. The total energy input depends on the total mass of the initial ${ }^{56} \mathrm{Ni}$ and the redistribution by transport effects. ${ }^{56} \mathrm{Ni}$ decays by electron capture which leads to the emission of high energy photons when the excited ${ }^{56} \mathrm{Co}$ transitions to its ground state. Besides decay via electron capture, $18 \%$ of all decays of ${ }^{56} \mathrm{Co}$ are $\beta^{+}$and lead to the emission of a positron.

Escape Probabilities: First, we want to discuss the role of the escape probabilities and their implications for LCs (Fig. 3). The small cross sections for $\gamma$ - rays result in rapidly increasing escape fractions. Already at maximum light, $\approx 20$ days after the explosion, $15 \%$ of all $\gamma$ s escape. This fraction increases to about 85 and $98 \%$ by day 100 and 300 , respectively. In contrast, the large positron cross sections cause almost complete local annihilation of positrons up to about 200 days. The length of the positron path depends on the size of $B$, which causes a strong dependence of the positron escape fraction on $B$ at later times. However, as the kinetic energy of positrons only account for some $3 \%$ of the total energy produced by ${ }^{56} \mathrm{Co}$ decay, the total energy input is dominated by $\gamma$ rays until around day $150-200$ (Fig. 

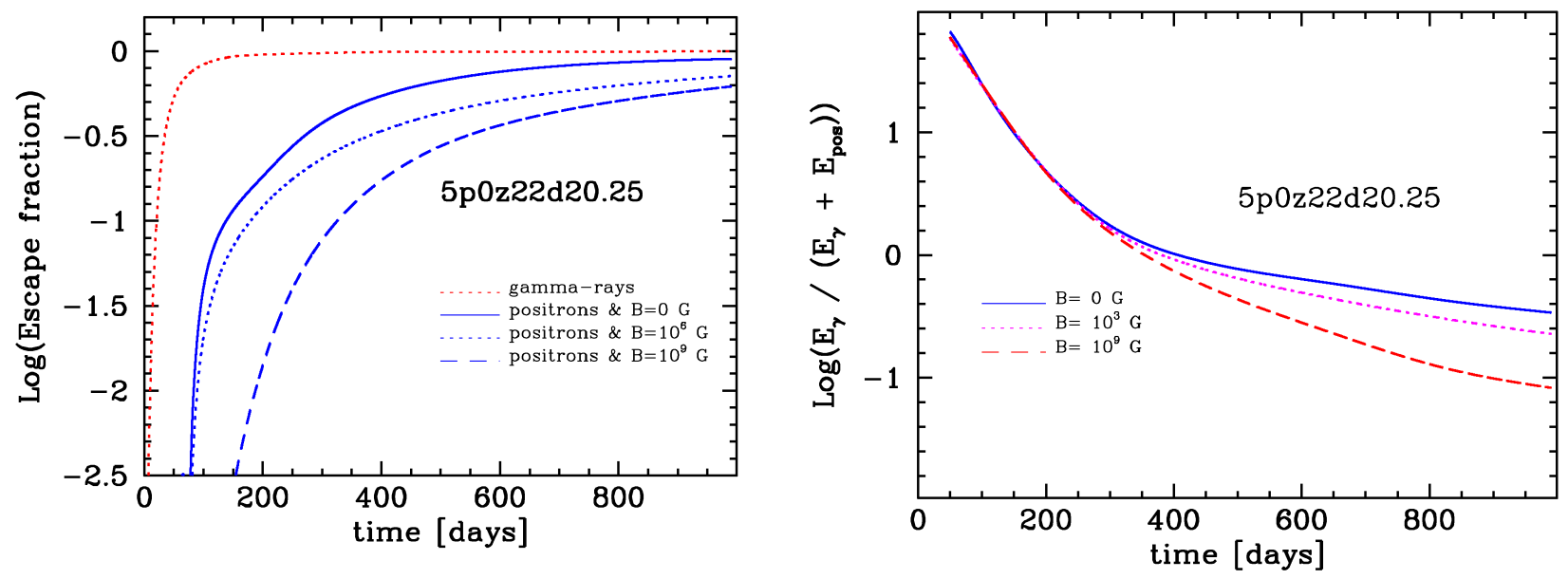

FIG. 3.- Escape probability for photons and positrons (left) and contribution to the total energy input in \% (right) as functions of time. Magnetic fields influence positron transport only. Probing magnetic fields requires late-time observations.

3). Thus studying the $B$ field via the influence of energy deposition on optical/IR wavelengths inherently requires late-time observations.

Light Curves: Previously, detailed simulations for positron transport effects on the bolometric light curves have been published by (Milne et al. 1999) for a wide range of models based on the deflagration model W7 (Nomoto 1984), the delayed-detonation models (Yamaoka et al. 1992; Hoeflich \& Khokhlov 1996; Hoeflich et al. 1998), helium detonations (Ruiz-Lapuente et al.|1993; Hoeflich \& Khokhlov 1996) and pulsating-delayed detonation and merger models (Hoeflich et al. 1995; Hoeflich \& Khokhlov 1996). We have compared the energy escape by positrons of our reference model and dipole fields with model DD23C (Hoeflich et al. 1998) and radial magnetic fields used in the sample of Milne et al. (1999). We find that our our escape fractions agree with Milne et al. (1999) within $\approx 20 \%$. For high $B$ fields, our escape fractions are slightly smaller because we include non-radial fields whereas Milne et al. (1999) consider the radial component only.

For a brief discussion, we use the V-Band (V) as a proxy for the bolometric LC (Arnett 1980). LCs are shown in Fig. 4 for low and high $B$ for dipole and turbulent fields. The scale of the turbulent field has been taken to be one pressure scale height in the progenitor WD. The formation of early-time optical and IR light curves have been previously discussed (Hoeflich et al. 1998; Hoeflich et al. 2002; Dessart et al. 2014).

Between 60 and 200 days, the decay of $\mathrm{V}$ is steeper than is expected from the decay of ${ }^{56} \mathrm{Co}$ because $\gamma$-ray photons dominate the energy input and they rapidly increase their escape probability, as shown in Fig. 3 . In fact, this discrepancy caused early predictions that SNe Ia are not powered by ${ }^{56} \mathrm{Ni}$ (Colgate et al. 1962). Note that the decline rate increases at about 100 to 200 days with decreasing brightness because more centrally concentrated ${ }^{56} \mathrm{Ni}$ causes a delay in the $\gamma$ escape (Hoeflich et al. 1993, 2002).

After $300+$ days, almost all $\gamma$ rays escape and the LC is dominated by positrons. As the envelope is now optically thin for optical photons the anisotropy effects are expected to be small. At $\approx 300$ days, $\mathrm{V}$ closely mimics the decay curve of cobalt. The change of slope after this point may be used to estimate $B$ (Milne et al. 1999). At about 300 days, the influence of $B$ remains small $\approx 0.05^{m}$. After about 3 years, the influence of $B$ grows to about one magnitude. Even for high $B$, the path of positrons follows the magnetic field line allowing some positron mobility and, then, positron escape. In contrast, for a turbulent field photons are trapped, which results in local energy deposition (see Fig. 4) until the field weakens to the point that the Lamour radius is larger than the size of the turbulence. Despite the significance of both the size and morphology of the $B$ field on late-time bolometric light curves, there are several limitations in using LCs to probe $B$. Monochromatic light curves are needed to reconstruct bolometric LC. However, V or UBVRI may not actually represent the bolometric light curves. It has been suggested that emission will shift from the V-Band to the infrared as the timescales of atomic transitions in the optical range increase relative to those in the infrared due to falling matter densities (called the 'IR catastrophe'). In models, the 'IR catastrophe' may set in anywhere from 300 to 400 days depending on the details of the atomic model and data, and the abundances (Fransson et al. 1996). Moreover, recent observations of SNe Ia identified mid-IR emission lines as major cooler starting at about 3-4 month after the explosion (Gerardy et al. 2007; Li et al. 2014). Finally, other energy sources may contribute to the energetics, such as interaction of the envelope with its surroundings, which will deposit energy in the high velocity layers. Moreover, the diversity among SNe Ia has been well established and variations in the ${ }^{56} \mathrm{Ni}$ distributions will change the bolometric light curves. These uncertainties and variables make it difficult to extract $B$ field strengths from just the one quantity provided by LCs.

The Energy Distribution: We want to shift our discussion to the distribution of the energy deposition and the resulting line profiles. The energy depositions by $\gamma$-rays and positrons and positrons only are given in Fig. 5. Up to about 100 to 200 days, the contribution from positrons is locally deposited due to high cross sections. Non-locality is produced by $\gamma$-rays. At early times, about a week after the explosion, the optical depth for $\gamma$-rays is still large and the energy input closely traces the distribution of the radioactive isotopes (Fig. 21). We can see the "hole" in the center 


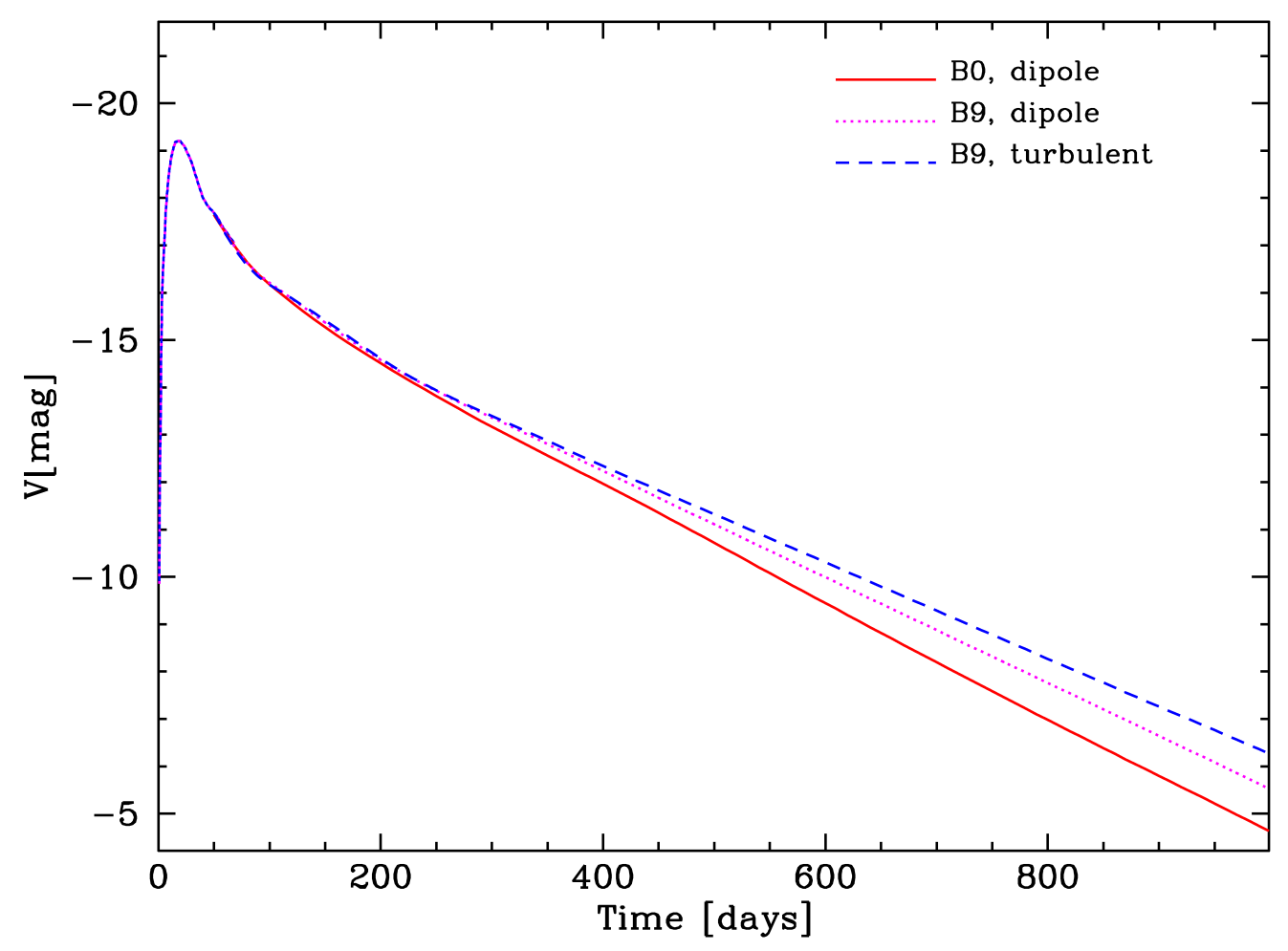

FIG. 4.- Influence of the size and morphology of $B$ on the visual light curves for our reference model. V is given for dipole and turbulent fields.
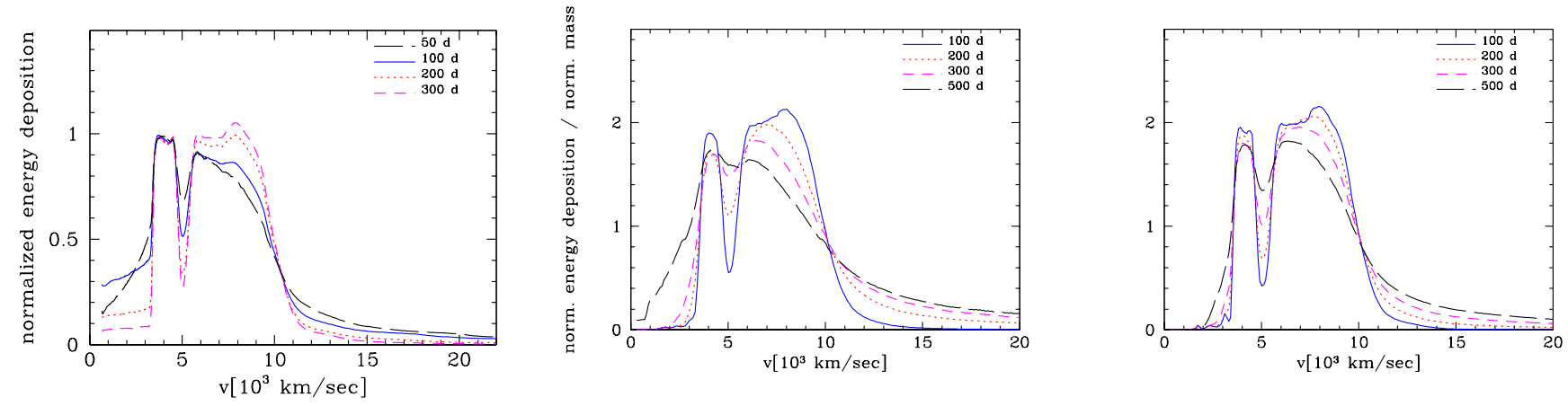

Fig. 5. - Energy input $\dot{E}$ per gram and second by both $\gamma$-rays and positrons assuming local trapping (left), and angle-averaged by positrons for initial magnetic fields of $B=1$ (middle) and $10^{9} \mathrm{G}$ (left), respectively. We normalized the total $\dot{E}$ to $v \approx 4000 \mathrm{~km} / \mathrm{sec}$ to compare the overall distributions and to compensate for the rapid decrease of trapped energy by $\gamma$-rays (see Fig. 3). $\dot{E}$ of positrons has been normalized to the average over the entire envelope.

occupied by stable, electron capture elements. Due to geometrical dilution, the optical depth for $\gamma$ photons decreases with time $(t)$ as $\approx t^{-2}$. This leads to an increasing energy deposition in the central region and larger heating of the outer layers. By about 100 days, the central heating peaks. With time, positrons take over as the main contributors to the energy deposition, and a central 'hole' reappears.

We start with the case of positron transport without $B$. Trapping can be attributed to the opacities only as the positron travels along a ray. The escape fractions and the normalized total and positron energy redistribution functions are given in Fig. 3 \& 5. Up to about 100 days, positrons continue to be locally confined. At 200 days, the outer layers are transparent, diffuse enough that the positrons escape, lowering the amount high velocity material is heated, but positrons in the inner layers remain locally confined. At 300 days small features like the dip in Ni at $5,000 \mathrm{~km} / \mathrm{sec}$ are starting to be washed out, and there is some penetration into the core. Note that dip in ${ }^{56} \mathrm{Ni}$ is not physical, but an artifact of spherical DDT. However, it is a good indicator to what degree positron transport has become non-local. After 500 days, the non-radiating core and the area around it is the only part of the envelope dense enough to have almost complete positron capture. At 300 to about 400 days, the total energy deposition is at 


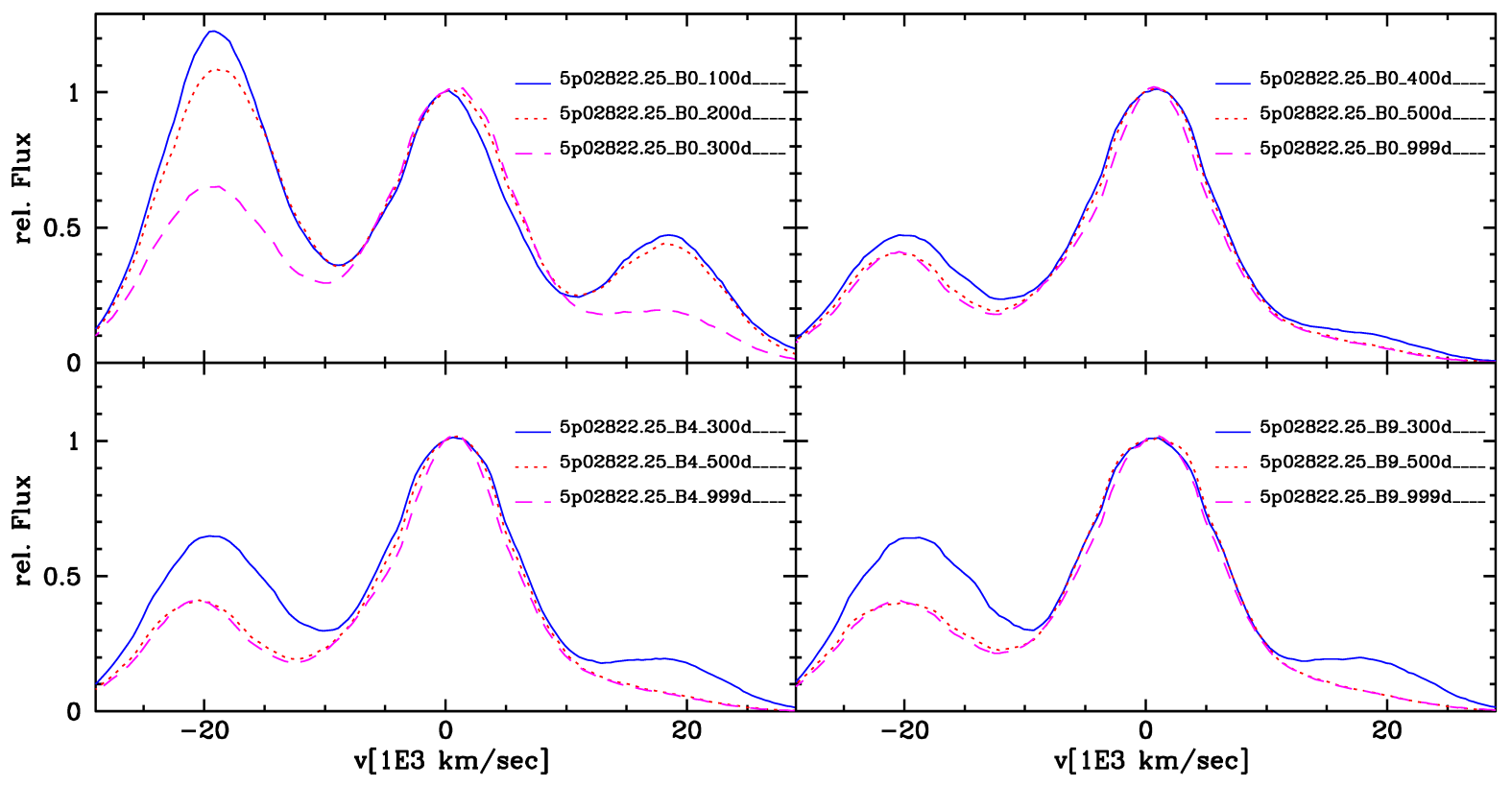

FIG. 6.- Evolution of the NIR for $B=1 G$, and $10^{4,9} G$ are shown in the upper and lower panels, respectively. The continuum flux has been subtracted and the flux normalized to the peak of the $1.644 \mu \mathrm{m}$ feature. The spectra are labeled by $A_{-} B b_{-} C d_{-} T_{-}$where $A$ identifies the model,$b=\log (B)$, and $C$ is the time after the explosion in days. $T=1,5$ and 3 denotes observation from polar and equatorial direction, respectively, and $T=\_$indicates angle averaging.

a minimum at the center and, subsequently, increases due to the growing mean free path of positrons.

Magnetic fields determine the path of positrons and thus introduce three more parameters into our discussion: The size of the field, its morphology, and the orientation of the $B$-field relative to an observer. The main effect of the $B$ field on the escape and energy redistribution can be understood in terms of the Lamour radius $r_{L}$ compared to the size of the envelope and any sub-structures considered. It increases the effective mean free path and influences the direction.

For dipole fields, we see that high magnetic fields delay the overall evolution of the energy distribution by about one year. For example, the distribution at 200 days resembles closely that of the high $B$ field at day 500 (Fig (5).

From the previous sections and within the delayed detonation scenario, we can conclude the following: Up to about 200 to 300 days, the energy deposition functions do not depend on magnetic fields because $\gamma$-ray transport dominate or positrons are deposited locally regardless of the field. Subsequently, positron transport effects may become important, depending on $B$.

\subsection{NIR Line Profiles for Dipole Fields}

The goal of this section is to demonstrate the signature of radiation and positron transport on late-time spectra. We will discuss in detail the $1.644 \mu \mathrm{m}$ spectral feature including its evolution with time, and its dependence on the orientation of the observer. In addition, adjoining strong NIR features are considered to demonstrate the impact of line blending and how blending may severely limit a kinematic interpretation of profiles. For the spectral band, we have calculated a full grid of 30 models for $B=10^{0,3,4,6,9} G$ at $100,200,300,400,500,999$ days with counters in 5 and 8 directions in the Eulerian $\Theta$ and $\phi$ coordinates. Here, we show only a few of the resulting 1200 spectra.

Our reference model, though spherical, requires full 3D-transport because of the axial symmetry introduced by the magnetic field. Chemical abundances take into account the variability caused by the radioactive decay ${ }^{56} \mathrm{Ni}^{56}$ $C o \rightarrow{ }^{56} \mathrm{Fe}$. The excitation balance has been calculated using our non-LTE code (Hoeflich et al. 2004) as a function of time. For the ionization, we found a mean ionization of about 1 to 1.5 electrons per atom, and have assumed a value of 1.5. We neglect second order effects on the excitation and ionization although the ionization in the central regions will depend on the heating by positrons and at late times, iron group elements may be neutral. As discussed in the final section, this approximation can be tested by observations but can be justified as follows: Lower ionization means a slightly larger mean free path, by about 20-30\% (Fig. 11). However, a larger mean free path implies more heating which, in turn, decreases positron transport (see Sect. 2) which, in turn, reduces the feedback effect. Tests have shown that the total effect on the spectral features remain small.

To discuss line profiles, the continuum fluxes have been subtracted and the maximum fluxes normalized. The actual abundances have been used from the model 5p0z22.25. For the spectral synthesis, we included the forbidden line profiles of [Fe II] at 1.533, 1.560, 1.644, 1.664, 1.677, 1.712, $1.745 \mu \mathrm{m}$ and [CoIII] of 1.55, 1.74, $1.76 \mu \mathrm{m}$. The continuum flux in the $1.6-1.7 \mu m$-band are $0.24,0.15,0.12,0.1,0.06$ and 0.02 at 100, 200,300, 400, 500 and 999 days, respectively.

The evolution with time is shown in Fig. 6 in velocity space corresponding to wavelengths between 1.5 and $1.8 \mu \mathrm{m}$. There are three prominent features at about $-20,000,0$ and $+18,000 \mathrm{~km} / \mathrm{sec}$. 


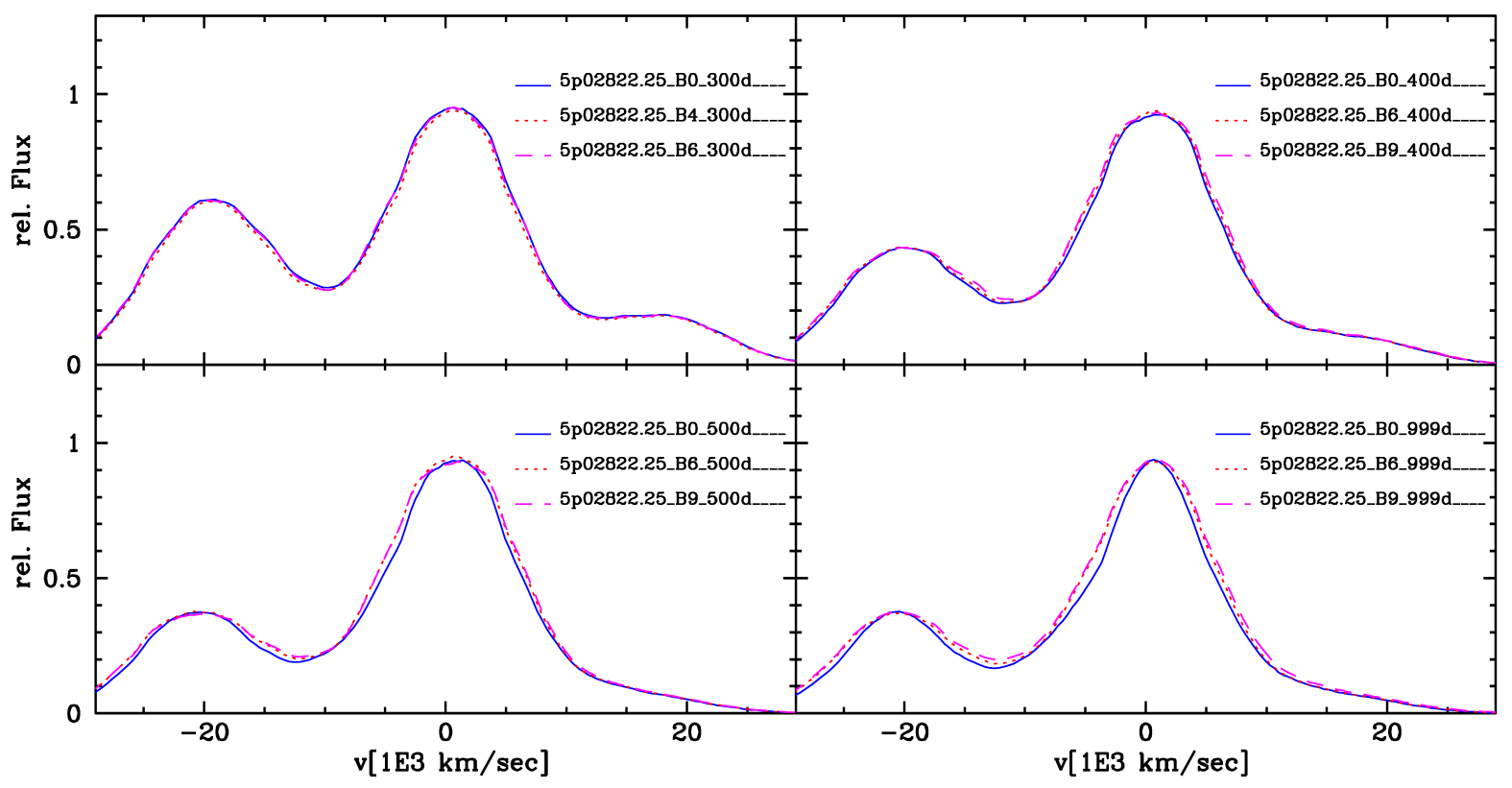

Fig. 7.- Influence of the size of the $B$ field on line profiles at 300, 400, 500 and 999 days. For the labeling scheme, see Fig. 6

Low Magnetic Fields: We start with a discussion of the angle averaged line profile of [Fe II] at $0 \mathrm{~km} / \mathrm{sec} \equiv 1.644 \mu \mathrm{m}$ because line-blending effects for this feature remain small except in the wings (Hoeflich et al. 2004). By day 100, the profile is peaked because gamma-rays are non-local and mostly absorbed in the central, high density region which has stable ${ }^{54} \mathrm{Fe}$. The [FeII] feature is asymmetric and blue-shifted by about $2000 \mathrm{~km} / \mathrm{sec}$ because the photosphere blocks the redshifted layers of the envelope. By day 200 , the blue shift is reduced to about $\approx 800 \mathrm{~km} / \mathrm{sec}$. With time, the feature becomes wider and reaches a maximum width at about 300 days. At around the same time it develops a 'flat-top' or 'stumpy' appearance in the center. As discussed and shown in Sect. 5, a caustic profile is produced if ${ }^{56} \mathrm{Ni}$ is homogeneously mixed to the center or the explosion of a low-density WD because the emission would follow the density structure (Hoeflich et al. 2004). By about day 200, positron transport results in increasingly wider line wings and a small low velocity emission component. By day 500, positrons are able to enter and deposit their energy in the core, and the profile becomes increasingly peaked.

Role of Magnetic Fields: Magnetic fields will hardly change line profiles up to about a year after the explosion. At about 300 days, the envelope is mostly optically thin in the 'quasi-continuum'. Observations of the profile of [FeII] at $1.644 \mu \mathrm{m}$ at this time provides an excellent tool to measure the distribution of ${ }^{56} \mathrm{Ni}$ in the velocity space. Earlier, optical depth effects in the continuum and the contribution of $\gamma$-rays are important contributors to the energy input. Later, positrons transport energy into the central regions, and at a rate dependent not just on the ${ }^{56} \mathrm{Ni}$ distribution and density structure, but also on the magnitude and structure of the $B$-field. In practice, early variability in the profiles allows us to separate optical depth and geometry effects, to probe the distribution of the central non-radioactive Fe and to test for ionization effects.

To detect the effects of $B$, we want to focus on the late time evolution (Fig. 7). With increasing $B$, the evolution from 'flat-topped' or 'stubby' to more centrally peaked profiles is delayed by about one year for high $B$ fields, as can be expected from the discussion above. Lines are narrower by about $20 \%$ by day 500 compared to models with $B>10^{6} G$. Observations at about 1000 days would allow the measurement of $\approx 10^{9} \mathrm{G}$.

Perils of Line Blending: The kinematic interpretation of emission features breaks down if line blending becomes important, as it is the case for features in the optical and most features in the NIR. The two features neighboring the $1.644 \mu \mathrm{m}$ line, at $-20,000$ and $+18,000 \mathrm{~km} / \mathrm{sec}$ in Fig. 6, are illustrations of the perils of apparently clean features. In fact, they are blends. The former feature is produced by transitions of [Fe II] 1.533, 1.560 and of [CoIII] at $1.55 \mu \mathrm{m}$. The latter feature is produced by transitions of [FeII] at 1.712, 1.745 and of [CoIII] at 1.74, $1.76 \mu \mathrm{m}$.

The evolution of both these 'satellite' features is dominated by the decay of radioactive ${ }^{56} \mathrm{Co}$. Up to about day 200 , the forbidden ${ }^{56} \mathrm{Co}$ lines are responsible for most of the emission. After about day 300 , only ${ }^{56} \mathrm{Fe}$ is left, as evidenced also by the slowly varying line ratio between the $1.644 \mu \mathrm{m}$ feature and the 'satellites'.

Early on, the red feature at $+18,000 \mathrm{~km} / \mathrm{sec}$ shows a blueshift similar to [Fe II] at $1.644 \mu \mathrm{m}$ but it is broader

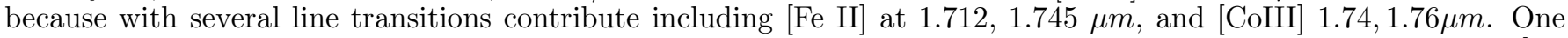
may expect that, with time, the feature would be shifted towards the blue because of the longer wavelength of the [Co III] transitions. However, we see a red-shift because the feature formed on the wing of the $1.644 \mu \mathrm{m}$ which produces a 'sloped' quasi-continuum.

The $-20,000 \mathrm{~km} / \mathrm{sec}$ feature at $\approx 1.55 \mu \mathrm{m}$ dominates the NIR spectra early on. However, due to its nature, i simple interpretation may be misleading. With time, this feature undergoes a blue shift opposite to the behavior of the [Fe 


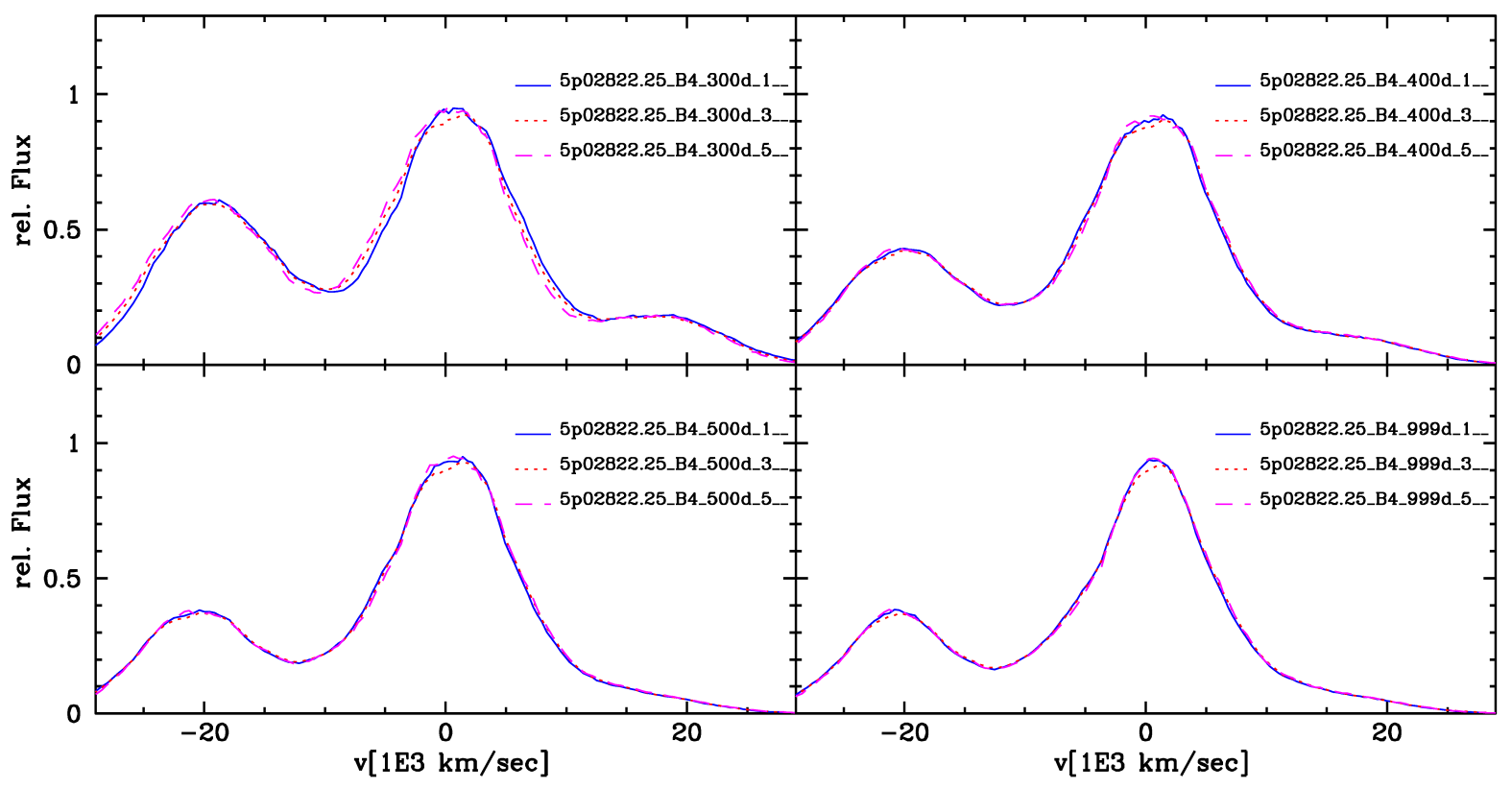

FIG. 8. - Directional dependence of the spectrum of the reference model as a function of the Doppler shift relative to the forbidden [FeII] at $1.644 \mu \mathrm{m}$. assuming a dipole field $10^{4} \mathrm{G}$. The solid, dotted and slashed lines correspond to $\Theta$ of 0,90 and 180 degrees. The panels show the spectra at $300,400,500$ and 999 days after the explosion.

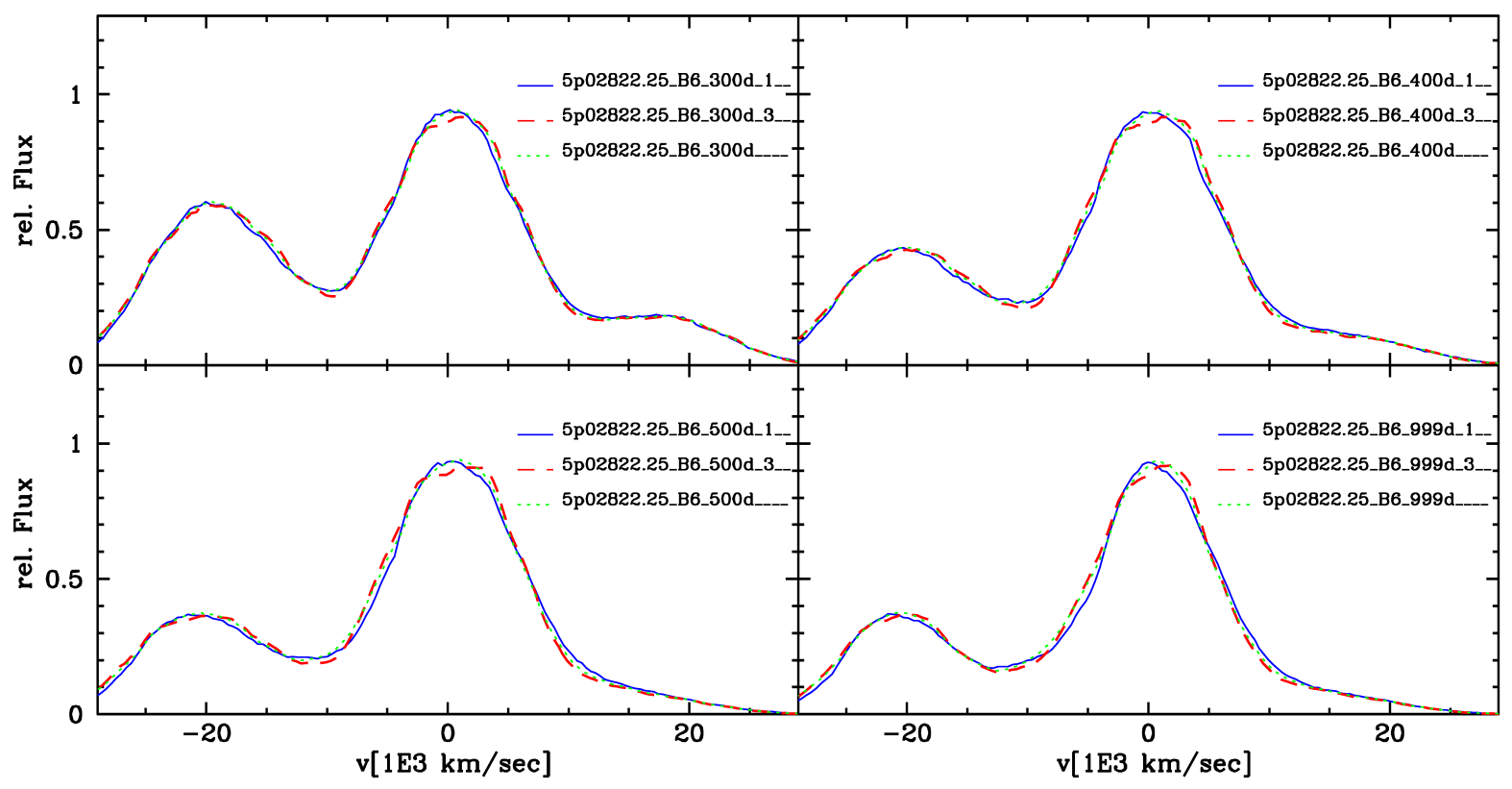

Fig. 9.- Same as fig. 8 but $B=10^{6} G$.

II] at $1.644 \mu \mathrm{m}$. Taking at face value and in a kinematic interpretation, this would mean that we see an off-set in ${ }^{56} \mathrm{Ni}$ whereas this shift, at least in the models, is due to the changing importance of [Co III] vs. [FeII].

In summary, time evolution allows us to detect and, potentially, to separate line blending, geometry and $B$ field effects. The [Fe II] feature at $1.644 \mu$ is rather unique in the NIR.

Directional Dependence of NIR Features: Large-scale magnetic dipole fields introduce a directional dependence of the observed line profile. The variations in the profiles can be understood in terms of the Lamour radius given in Tab. 1. We consider only times later than 300 days when positron transport effects may be significant. For low $B$ fields, the Lamour radius is much larger than the envelope and positrons travel on rays which results in isotropic profiles.

In Figs. $9 \&$ 10, the directional dependence of profiles are shown for high magnetic fields. For $B=10^{9} G$, the positrons follow the field lines with an increase in the 'effective' cross section. Variations in the energy deposition 


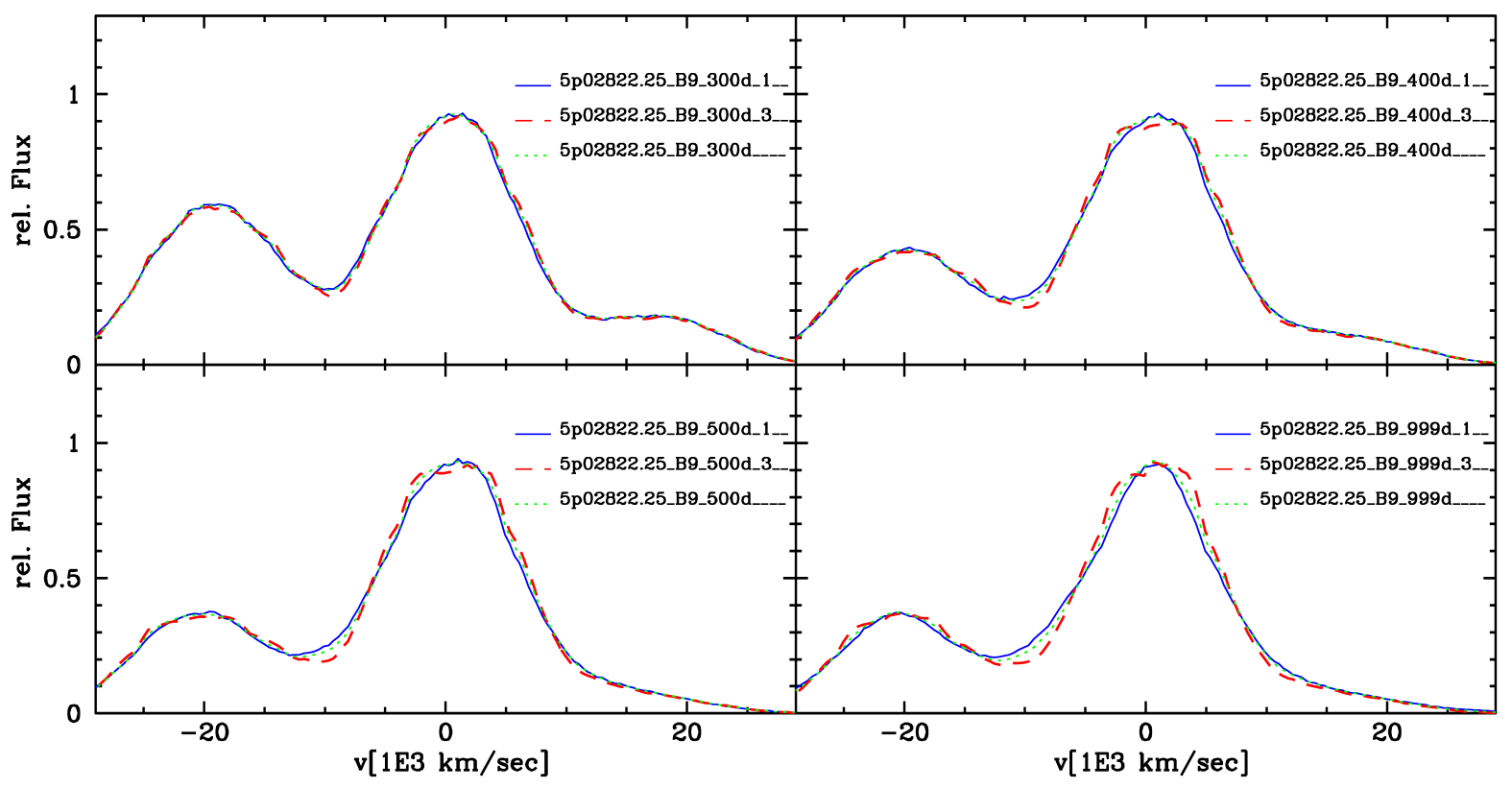

FIG. 10.- Same as fig. 8 but $B=10^{9} \mathrm{G}$.

and, thus, the energy deposition depends mainly on the mean free path. By day 1000, positrons created in the polar regions can travel to the center regardless of the $B$ field. More generally, positrons created at more equatorial latitudes are funneled towards the pole. This geometry effect causes maximum directional dependence. From the pole, we see flat-topped profiles with an increased line width. Seen from the equator, late time profiles are centrally peaked.

Lowering the $B$ field increases the mean free path, and the path of the positron is bent but does not exactly follow the field lines. For $B=10^{6} G$, the directional dependence shows up earlier. High precision observations of the time dependence of profiles would allow us to learn about the orientation of the field relative to the observer.

\subsection{Influence of the Morphology of the Magnetic Field}

This leads us to the effects of the morphology of the $B$ fields. We may expect a dipole field in a WD prior to the runaway but it may be distorted by the turbulent motion during the thermonuclear runaway, or by RT-instabilities during the explosion of the WD.

As an example and limiting case we have calculated the transport for a turbulent field. In calculations of the thermonuclear runaway, the largest turbulent eddies are $\approx 5 \%$ of the WD (Hoeflich \& Stein 2002). We note that the energy deposition is close to isotropic.

The results are dominated by the change of the gyro-radius relative to the size of the structure of the $B$ field (Tab. 1). $B$ in excess of $10^{6} \mathrm{G}$ results in local trapping of positrons with some transport effects starting at about 500 to 1000 days. For fields less than $\approx 10^{4} G$, the gyro-radius is much larger than the size of the envelope and, consequently, the results are identical to the angle-averaged profiles in Figs. 6] \& 7 . Probing the magnetic fields require time series of profiles after about 2 to 3 years, or magnetic fields between $10^{4 \cdots 6} G$ observed beyond 400 days. As discussed above for very high magnetic fields, LCs come close to the case of full trapping.

\section{DISCUSSION AND CONCLUSIONS}

We have presented a new implementation of positron transport using a Monte Carlo method. This allows us to the study of transport effects in full 3D for arbitrary magnetic fields without the need for either the low or large field approximations.

In SNe Ia, light curves and spectra are powered by the radioactive decay from ${ }^{56} \mathrm{Ni} \rightarrow{ }^{56} \mathrm{Co} \rightarrow{ }^{56} \mathrm{Fe}$. The evolution of the energy deposition is dominated by a transition from the $\gamma$-ray to the positron dominated regime between 200 and 300 days, and the changing optical depth due to the expansion. Because positron transport depends on $B$, late time observations provide a tool to probe the fields.

Our study is based on the explosion of a $M_{C h}$ mass WD, specifically a delayed detonation model for a Branch-normal SN Ia, with $B$ fields imprinted at the time of the explosion. Up to about 200 days after the explosion, positron transport can be neglected. By 300 days, positron transport becomes important and changes both the total and redistribution of energy from radioactive decay.

The effect of $B$ on the late time light curve grows from $\approx 0.1^{m}$ at day 300 to $\approx 1^{m}$ some three years after the explosion. We found LC variations due to $B$ comparable to previous studies within $\approx 20 \%$ (Milne et al. 1999). Differences can be attributed to the magnetic field structure assumed. Milne and previous authors considered radial magnetic fields whereas we assumed that the $B$-field is frozen into the expanding ejecta. Our assumption is justified because the expansion is homologous. The additional component in $B$ results in a larger degree of trapping. In practice, however, 
the use of LCs may be limited. It relies on monochromatic V and UBVRI LCs to reconstruct the bolometric LC (Sect. 4). Photon redistribution from the visual to the mid- and far-IR becomes increasingly important starting several months after the explosion and, after a few years, may result in an 'IR-catastrophe'. An additional problem may be related to the fact that LCs measure the total energy input. Additional energy contributors may become important, such as interaction with the circumstellar medium. Moreover, the diversity in SNe Ia may further limit the use of differential analysis based on a single quantity.

The use of late-time line profiles of iron group elements overcomes some of these problems. The formation of these lines depends on the population of the upper atomic level and, thus, profiles are hardly effected by the overall photon redistribution or e.g. ongoing interactions which deposit energy in the outer rather than the ${ }^{56} N i$ layers. Line profiles are a measure of the redistribution function of the input energy in velocity space rather than the escape fraction.

We have demonstrated that magnetic fields can be probed by the line shape and its evolution. Line blending due to Doppler shift is a major problem in SNe Ia but we have identified as a suitable spectral feature the forbidden [Fe II] line at $1.644 \mu \mathrm{m}$. This feature shows only minor blending and thus allows a kinematic interpretation of the profile if optical depth effects are taken into account. Other strong, individual NIR (and optical) features are of limited use because they originate from strong blends, mostly of [Fe II] and [CoIII] lines.

We find that large scale, dipole fields have the biggest effect on the line profile. Up to about 200-250 days after the explosion, positrons are mostly locally trapped and, consequently, the profile depends on the chemical and density structure of the explosion models. The change with time of the feature can be understood by the change from the regime of energy input by $\gamma$-rays to that of positrons. Line profiles depend on the geometry and distribution of ${ }^{56} \mathrm{Ni}$ and the density structure of the explosion model (Hoeflich et al. 2004; Motohara et al. 2006; Hoeflich 2006; Maeda et al. 2011). At early times, profiles are peaked because of excitation due to non-local $\gamma$-rays which, with time, become increasingly 'flat-topped' until non-local positrons can excite the low velocity iron-group elements.

To produce 'flat-topped' profiles at day 300-400 as have been observed in SN2003du and SN2004hv, we need magnetic fields of $10^{6} G$ or larger. For large-scale dipole fields, the supernovae must be seen from equatorial directions. Seen from an angle close to the pole, the profile is broad ('stumpy'), but with a 'round-top' because positrons can travel towards the central region parallel to the magnetic field.

To separate optical depth effects of the envelope, ionization and orientation effects, time series of the NIR are crucial. Spectra are needed with a resolution of, at least, 300 to $500 \mathrm{~km} / \mathrm{sec}$ between 300 to 400 days, and a signal to noise ratio of 10 to $20 \%$, or a signal to noise of 20 to $30 \%$ after 500 days.

We discussed the influence of the morphology of the B-field. For magnetic fields of turbulent scales, the profiles will be non-directionally dependent, and somewhat smaller $B$ fields are sufficient. To separate various morphologies of $B$, the typical mean free path of positrons must be larger than the characteristic structures, which would require spectra beyond day 400. These effects are currently under investigation.

Our simulations suggest the need of $B$ in excess of $10^{6} G$ to explain persistent 'flat-topped' or 'stumpy' profiles as observed for SN2003du and SN2003hv after about a year. As discussed below and in Hoeflich et al. (2013), fields of this size will affect the hydrodynamics of the smoldering phase of the WD just prior to the runaway and, possibly, deflagration burning. This is the first strong, observational evidence that magneto-hydrodynamical effects should be taken into account during the runaway and deflagration phase of SNe Ia. We also note that explosion models with central densities less than $10^{9} \mathrm{~g} / \mathrm{cm}^{3}$ will give ${ }^{56} \mathrm{Ni}$ up to the center and produce narrow overall profiles consistent with the 'caustic' density profile of SNe Ia (see Fig 2) as discussed in Hoeflich et al. (2004); Stritzinger et al. (2014). Thus, SN2003du and SN2003hv are likely to originate from $M_{C h}$ mass explosions with very limited amount of mixing of ${ }^{56} \mathrm{Ni}$ during the explosion.

However, SNe Ia are a diverse group of objects as discussed in the introduction. Positron transport effects depend on the free mean path, thus, the density and $B$. The time of importance will depend on the overall ${ }^{56} N i$ distribution. In Fig. 11, we show the evolution of our reference model but with central ${ }^{56} \mathrm{Ni}$. The late time profiles are 'peaked' rather than 'flat-topped' or 'stubby' unlike our reference model. Because of the higher densities, positron transport effects remain small and evolution starts at about 3 years rather than 1 to 1.5 years. The [Fe II] feature shows little evolution between 200 days and about 3 years after the explosion. This kind of profiles may be produced either by lower central densities of the WD and/or mixing during the explosion. The distribution of ${ }^{56} \mathrm{Ni}$ is important and line profiles will allow us to decipher both $B$ fields and the structure.

In this context, SN 2014J in M82 Fossey et al. (2014) may shed new light on SNe Ia. It is the first SNe Ia for which $\gamma$ rays have been detected. These are consistent with a ${ }^{56} \mathrm{Ni}$ production of $0.6 M_{\odot}$ (Isern et al. 2014; Churazov et al. 2014). Recently, (Diehl et al. 2014) analysed the early ${ }^{56} \mathrm{Ni}$ lines at 158 and $812 \mathrm{MeV}$ in SN 2014J. The authors report ${ }^{56} \mathrm{Ni}$ lines at 158 and $812 \mathrm{MeV}$ with a line width of $\approx 5000 \mathrm{~km} / \mathrm{sec}$ and which are not shifted in rest-frame of the supernovae. The line fluxes require about $0.06 M_{\odot}$ of ${ }^{56} \mathrm{Ni}$. (Diehl et al. 2014) suggest a configuration in which helium is accreted in a belt around the progenitor seen 'pole-on'. During the explosion, parts of the He may be burned to ${ }^{56} \mathrm{Ni}$. If confirmed, this detection clearly demonstrates the diversity in ${ }^{56} \mathrm{Ni}$ distributions. In framework of this study, we may expect a narrow component on top of broader un-blended features in late-time NIR and MIR spectra. Because of the lower densities, we expect positron transport effects starting earlier for the high velocity component at about 100 to 200 days depending on B. For SN2014J, corresponding observations during the next few years are scheduled which will allow a $\mathrm{S} / \mathrm{N}$ at the level required. Note that an $0.1^{\mathrm{m}}$ effect on the bolometric is unlikely to be detected by light curves.

Finally, we have to emphasize the limitations of this study: we neglected the feedback of positron transport on the 


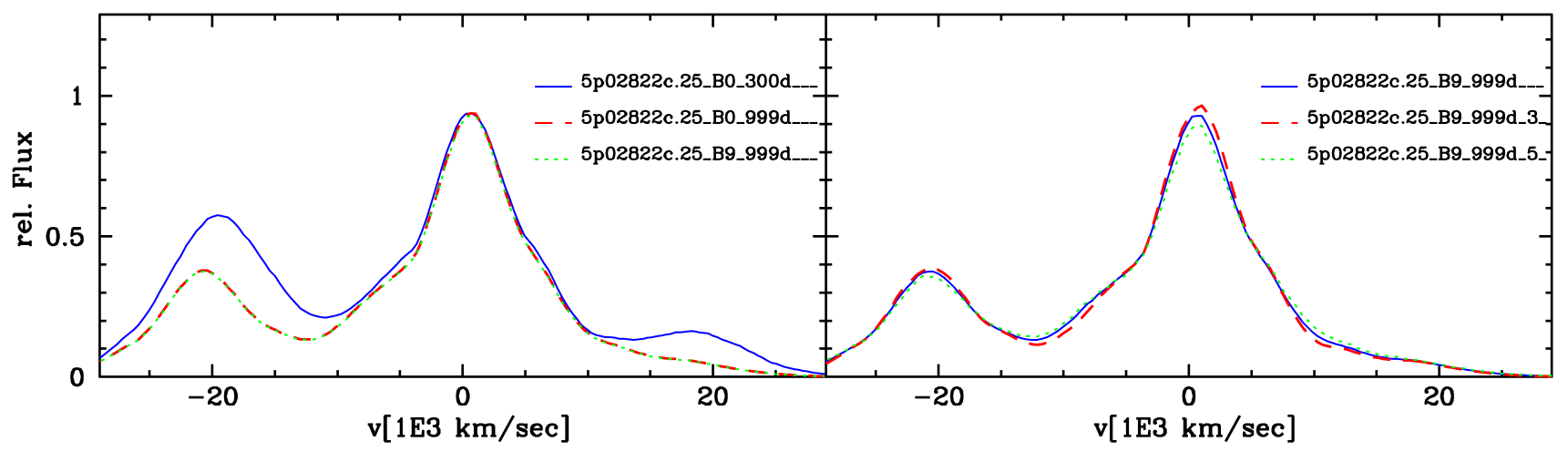

FIG. 11. - NIR profile of our reference model but assuming homogeneous mixing of ${ }^{56} \mathrm{Ni}$ down to the center. The average profile of the $1.644 \mu \mathrm{m}$ feature shows little evolution between 300 and 999 days other than due to the blends in the line wings (left). The profile is peaked rather than 'flat topped' or 'stumpy'. On the right, the average profile is compared to the polar and equatorial profiles for $10^{9} G$. By day 999 and high magnetic and dipole fields, the profiles have directional dependence.

ionization structure. Namely, we may have central neutral iron group elements Liu et al. (1997b); Jefferv et al. (1998); Liu et al. (1998) which can produce flat-topped profiles as ionization effect. However, the location of ionization fronts will be time-dependent and hardly produce persistent characteristics.

Our study suggests high initial magnetic fields and $M_{C h}$ WD for several SNe Ia. However, we lack time-series needed for more detailed analyses. Currently, a time series of SN2005df is being analyzed as part of the PhD thesis of Tiara Diamond. Obviously, we need more and time sequences in the NIR and this kind of observations will become more common in the near future. We will observe the nearby SN 2014J over the next few years to obtain S/N of a few percent, and corresponding are scheduled at Gemini. JWST, GMT, ELT and WFIRST will allow us to obtain NIR spectra on a similar or better S/N level for SNe Ia up to about Coma distance.

For turbulent magnetic fields, the quantitative results will depend on the turbulence spectrum present in the WD or created during the deflagration phase of burning. Currently, we study the influence of more realistic turbulent fields, and their influence on line profiles. Another question is related to the origin of high $B$ fields. WDs are observed with $B$-fields up to several times $10^{7} \mathrm{G}$, but the majority have no measurable B field (Liebert et al. 2003; Schmidt et al. 2003; Silvestri et al. 2007; Tout et al. 2008). Large $B$-fields may be produced either during the accretion phase, right before the thermonuclear runaway, or the deflagration phase. In a related project, we are currently studying properties of magnetic fields in the thermonuclear runaway: the growth of the field by dynamo action; impact of magnetic fields on velocity statistics; the suppression of the Rayleigh-Taylor instability by magnetic tension; and the alteration of flame speeds by anisotropic conduction. In three dimensional studies, there is a tendency for magnetic fields to increase the rate of growth in modes parallel to the field, due to the suppression of secondary instabilities, and a decrease in growth in modes transverse to the magnetic field (Stone \& Gardiner 2007b a; Ghezzi et al. 2004) which, eventually, may lead to an answer why we do not see RT instabilities to the extent predicted.

Above, we have discussed the limitations of light curve with respect to $B$. A combination of line profiles and LCs may help to overcome some of the problems with using LCs, in particular, if combined with observations of SNe Ia in the mid-IR to calibrate the photon redistribution effects. We are currently extending our LC studies along this path.

We would like to thank many colleges and collaborators for their support, in particular, E. Baron, D. Collins, T. Diamond, R. Fesen, A. Hamilton, E. Hsiao, A. Khokhlov, J. Maund, M. Phillips, D. Rubin, M. Stritzinger, N. Suntzeff, C. Telesco, S. Valenti, L. Wang, J.C. Wheeler and many observers to provide insights and data. The work presented in this paper has been supported by the NSF projects AST-0708855, "Three-Dimensional Simulations of Type Ia Supernovae: Constraining Models with Observations" and AST-1008962, "Interaction of Type Ia Supernovae with their Environment". In parts, the results presented have been obtained in course of a PhD thesis by R. Penney at Florida State University.

\section{REFERENCES}

Ambwani, K., \& Sutherland, P. 1988a, ApJ, 325, 820

- . 1988b, ApJ, 325, 820

Arnett, W. D. 1980, ApJ, 237, 541

Baron, E., Hoeflich, P., Krisciunas, K., et al. 2012, ApJ, 753, 105

Benz, W., Cameron, A. G. W., Press, W. H., \& Bowers, R. L. 1990, ApJ, 348, 647

Bowers, E., et al. 1997, MNRAS, 290, 663

Branch, D., \& Miller, D. L. 1993, Astrophysical Journal, Letters, 405, L5

Brown, B. L., \& Leventhal, M. 1987, ApJ, 319, 637

Burrows, A., \& The, L.-S. 1990, ApJ, 360, 626

Bussard, R. W., Ramaty, R., \& Drachman, R. J. 1979, ApJ, 228, 928
Calder, A. C., Krueger, B. K., Jackson, A. P., \& Townsley, D. M. 2013, Frontiers of Physics, 8, 168

Chan, K. W., \& Lingenfelter, R. E. 1991, ApJ, 368, 515

Chan, K.-W., \& Lingenfelter, R. E. 1993, ApJ, 405, 614

Chen, F. 2003, Introduction to Plasma Physics and Controlled Fusion (New York, NY: Plenum Press)

Churazov, E., Sunyaev, R., Isern, J., et al. 2014, ArXiv e-prints

Colgate, S., Petschek, A., \& Kriese, J. 1980, ApJ, 237

Colgate, S. A., Grasberger, W. H., \& White, R. H. 1962, Journal of the Physical Society of Japan Supplement, 17, C157

Dessart, L., Blondin, S., Hillier, D. J., \& Khokhlov, A. 2014,

MNRAS, 441, 532

Diehl, R., Siegert, T., Hillebrandt, W., et al. 2014, ArXiv e-prints 
DiStephano, R., \& Orio, M. 2012, in IAU Symposium, Vol. 281, Binary Paths to SNeIa Explosions, ed. R. DiStephano and M. Orio

Edwards, Z. I., Pagnotta, A., \& Schaefer, B. E. 2012, ApJl, 747, L19

Fesen, R. A., Hoeflich, P. A., Hamilton, A. J. S., et al. 2007, ApJ, 658,396

Fisher, A., Branch, D., Nugent, P., \& Baron, E. 1997, ApJl, 481, L89

Fossey, J., Cooke, B., Pollack, G., Wilde, M., \& Wright, T. 2014, Central Bureau Electronic Telegrams, 3792, 1

Fransson, C., Houck, J., \& Kozma, C. 1996, in IAU Colloq. 145: Supernovae and Supernova Remnants, ed. R. McCray \& Z. Wang, 211

Gamezo, V. N., Khokhlov, A. M., \& Oran, E. S. 2005, ApJ, 623, 337

Gamezo, V. N., Khokhlov, A. M., Oran, E. S., Ctchelkanova, A. Y., \& Rosenberg, R. O. 2003, Science, 299, 77

Gerardy, C. L., Meikle, W. P. S., Kotak, R., et al. 2007, ApJ, 661,995

Ghezzi, C. R., de Gouveia Dal Pino, E. M., \& Horvath, J. E. 2004, MNRAS, 348, 451

González Hernández, J. I., Ruiz-Lapuente, P., Filippenko, A. V., et al. 2009, ApJ, 691, 1

Gould, R. J. 1971, Physica, 60, 145

Greiner, J., Hasinger, G., \& Kahabka, P. 1991, A\&A, 246, L17

Guidry, M., \& Messer, B. 2013, Frontiers of Physics, 8, 111

Hoeflich, P. 2002, NAR, 46, 475

—. 2006, Nuclear Physics A, 777, 579

Hoeflich, P., Dragulin, P., Mitchell, J., et al. 2013, Frontiers of Physics, 8, 144

Hoeflich, P., Gerardy, C. L., Fesen, R. A., \& Sakai, S. 2002, ApJ, 568,791

Hoeflich, P., Gerardy, C. L., Marion, H., \& Quimby, R. 2006, New A Rev., 50, 470

Hoeflich, P., Gerardy, C. L., Nomoto, K., et al. 2004, ApJ, 617, 1258

Hoeflich, P., \& Khokhlov, A. 1996, ApJ, 457, 500

Hoeflich, P., Khokhlov, A., \& Mueller, E. 1994, ApJS, 92, 501

Hoeflich, P., Khokhlov, A., Wheeler, J. C., et al. 1996, ApJl, 472, L81

Hoeflich, P., Khokhlov, A. M., \& Wheeler, J. C. 1995, ApJ, 444, 831

Hoeflich, P., Müller, E., \& Khokhlov, A. 1993, A\&A, 97, 221

Hoeflich, P., \& Stein, J. 2002, ApJ, 568, 779

Hoeflich, P., Wheeler, J. C., \& Thielemann, F.-K. 1998, ApJ, 495, 617

Hoflich, P. 1995, ApJ, 443, 89

Howell, A., Hoeflich, P., Wang, L., \& Wheeler, J. C. 2001, ApJ, 556

Hoyle, F., \& Fowler, W. A. 1960, ApJ, 132, 565

Iben, Jr., I., \& Tutukov, A. V. 1984, ApJs, 54, 335

Isern, J., Hernanz, M., \& José, J. 2011, in Lecture Notes in Physics, Berlin Springer Verlag, Vol. 812, Lecture Notes in Physics, Berlin Springer Verlag, ed. R. Diehl, D. H. Hartmann, \& N. Prantzos, 233-308

Isern, J., Knoedlseder, J., Jean, P., et al. 2014, The Astronomer's Telegram, 6099, 1

Jeffery, D. J., Krstic, P. S., Liu, W., Schultz, D. R., \& Stancil, P. C. 1998, in Laboratory Space Science Workshop, 101

Kahabka, P., \& van den Heuvel, E. P. J. 1997, ARA\&A, 35, 69

Kasen, D., Röpke, F. K., \& Woosley, S. E. 2009, Nature, 460, 869

Kerzendorf, W. E., Schmidt, B. P., Asplund, M., et al. 2009, ApJ, 701,1665

Khokhlov, A. M. 1991, aap, 245, L25

-. 1995, ApJ, 449, 695

Kurucz, R. 1993, CDROM No. 1: Atomic Data for Opacity Calculations, SAO Cambridge, MA

Kurucz, R. L. 2002, in American Institute of Physics Conference Series, Vol. 636, Atomic and Molecular Data and Their Applications, ed. D. R. Schultz, P. S. Krstic, \& F. Ownby, $134-143$

Lang, K. R. 1999, Astrophysical Formulae (Berlin: Springer)

Lentz, E. J., Baron, E., Branch, D., \& Hauschildt, P. H. 2001 , ApJ, 557, 266

Li, D., Telesco, C. M., Alvarez, C., et al. 2014, The Astronomer's Telegram, 6018, 1
Liebert, J., Bergeron, P., \& Holberg, J. 2003, aj, 125, 348

Liu, W., Jeffery, D. J., \& Schultz, D. R. 1997a, ApJ, 483, L107

-. 1997b, ApJ, 483, L107

-. 1998, ApJ, 494, 812

Livne, E. 1999, ApJl, 527, L97

Lorén-Aguilar, P., Isern, J., \& García-Berro, E. 2009, A\&A, 500, 1193

Maeda, K., Mazzali, P. A., Deng, J., et al. 2003, ApJ, 593, 931

Maeda, K., Leloudas, G., Taubenberger, S., et al. 2011, MNRAS, 413, 3075

Marion, G. H., Hoeflich, P., Gerardy, C. L., et al. 2009, AJ, 138, 727

Maund, J. R., Hoeflich, P., Patat, F., et al. 2010, ApJl, 725, L167

Milne, P., The, L.-S., \& Leising, M. 1999, ApJS, 124, 503

Milne, P. A., Hungerford, A. L., Fryer, C. L., et al. 2004, ApJ, 613,1101

Mochkovitch, R., \& Livio, M. 1990, A\&A, 236, 378

Motohara, K., Maeda, K., Gerardy, C. L., et al. 2006, ApJl, 652, L101

Nadyozhin, D. 1994, ApJS, 92, 527

Niemeyer, J. C., \& Hillebrandt, W. 1995, ApJ, 452, 769

Nomoto, K. 1984, ApJ, 277, 791

Nugent, P., Baron, E., Branch, D., Fisher, A., \& Hauschildt, P. 1997, ApJ, 485, 812

Nugent, P. E. 1997, PhD thesis, THE UNIVERSITY OF OKLAHOMA, Norman Campus

Nussbaumer, H., \& Storey, P. J. 1988a, A\&A, 193, 327

-. 1988b, A\&A, 200, L25

Pakmor, R., Hachinger, S., Röpke, F. K., \& Hillebrandt, W. 2011, A\&A, 528, A117

Patat, F., Hoeflich, P., Baade, D., et al. 2012, A\&A, 545, A7

Penney, R. 2011, Master's thesis, Florida State University

Phillips, M. M. 2012, PASA, 29, 434

Piersanti, L., Gagliardi, S., Iben, Jr., I., \& Tornambé, A. 2003,

ApJ, 598, 1229

Plewa, T. 2007, ApJ, 657, 942

Poludnenko, A. Y., Gardiner, T. A., \& Oran, E. S. 2011, Physical Review Letters, 107, 054501

Quimby, R., Hoeflich, P., Kannappan, S. J., et al. 2006, ApJ, 636, 400

Rappaport, S., Chiang, E., Kallman, T., \& Malina, R. 1994, ApJ, 431,237

Rasio, F. A., \& Shapiro, S. L. 1994, ApJ, 432, 242

Reinecke, M., Hillebrandt, W., \& Niemeyer, J. C. 1999, A\&A, 347,739

Röpke, F. K., Gieseler, M., Reinecke, M., Travaglio, C., \& Hillebrandt, W. 2006, A\&A, 453, 203

Röpke, F. K., Kromer, M., Seitenzahl, I. R., et al. 2012, ApJl, 750, L 19

Ruiz-Lapuente, P., Jeffery, D. J., Challis, P. M., et al. 1993, Nature, 365, 728

Ruiz-Lapuente, P., Comeron, F., Méndez, J., et al. 2004, Nature, 431, 1069

Sadler, B. 2012, Master's thesis, Florida State University

Saio, H., \& Nomoto, K. 1985, A\&A, 150, L21

Saio, H., \& Nomoto, K. 1998, ApJ, 500, 388

Schaefer, B. E., \& Pagnotta, A. 2012, Nature, 481, 164

Schmidt, G. D., Harris, H. C., Liebert, J., et al. 2003, ApJ, 595, 1101

Segretain, L., Chabrier, G., \& Mochkovitch, R. 1997, ApJ, 481, 355

Shen, K. J., Bildsten, L., Kasen, D., \& Quataert, E. 2011, ApJ, in press, astro-ph/1108.4036

Silvestri, N. M., Lemagie, M. P., Hawley, S. L., et al. 2007, AJ, 134,741

Sim, S. A., Seitenzahl, I. R., Kromer, M., et al. 2013, MNRAS, 436,333

Stone, J. M., \& Gardiner, T. 2007a, Physics of Fluids, 19, 094104

-. 2007b, ApJ, 671, 1726

Stritzinger, M. D., Hsiao, E., Valenti, S., et al. 2014, A\&A, 561, A146

Tout, C. A., Wickramasinghe, D. T., Liebert, J., Ferrario, L., \& Pringle, J. E. 2008, MNRAS, 387, 897

van den Heuvel, E. P. J., Bhattacharya, D., Nomoto, K., \&

Rappaport, S. A. 1992, A\&A, 262, 97

Wang, B., Chen, X., Meng, X., \& Han, Z. 2009a, ApJ, 701, 1540 
Wang, B., Meng, X., Chen, X., \& Han, Z. 2009b, MNRAS, 395, 847

Wang, L., Hoeflich, P., \& Wheeler, J. C. 1997, ApJ, 483, L29

Webbink, R. F. 1984, ApJ, 277, 355

Wheeler, J. C., Hoeflich, P., Harkness, R. P., \& Spyromilio, J. 1998, ApJ, 496, 908

Whelan, J., \& Iben, I. J. 1973, ApJ, 186, 1007

Woosley, S. E., \& Weaver, T. A. 1986, in NATO ASIC Proc. 163:

Nucleosynthesis and its Implications on Nuclear and Particle

Physics, ed. J. Audouze \& N. Mathieu, 145-166
Woosley, S. E., \& Weaver, T. A. 1994, ApJ, 423, 371

Yamaoka, H., Nomoto, K., Shigeyama, T., \& Thielemann, F.-K. 1992, ApJ, 393, L55

Yoon, S.-C., Podsiadlowski, P., \& Rosswog, S. 2007, MNRAS, 380,933 


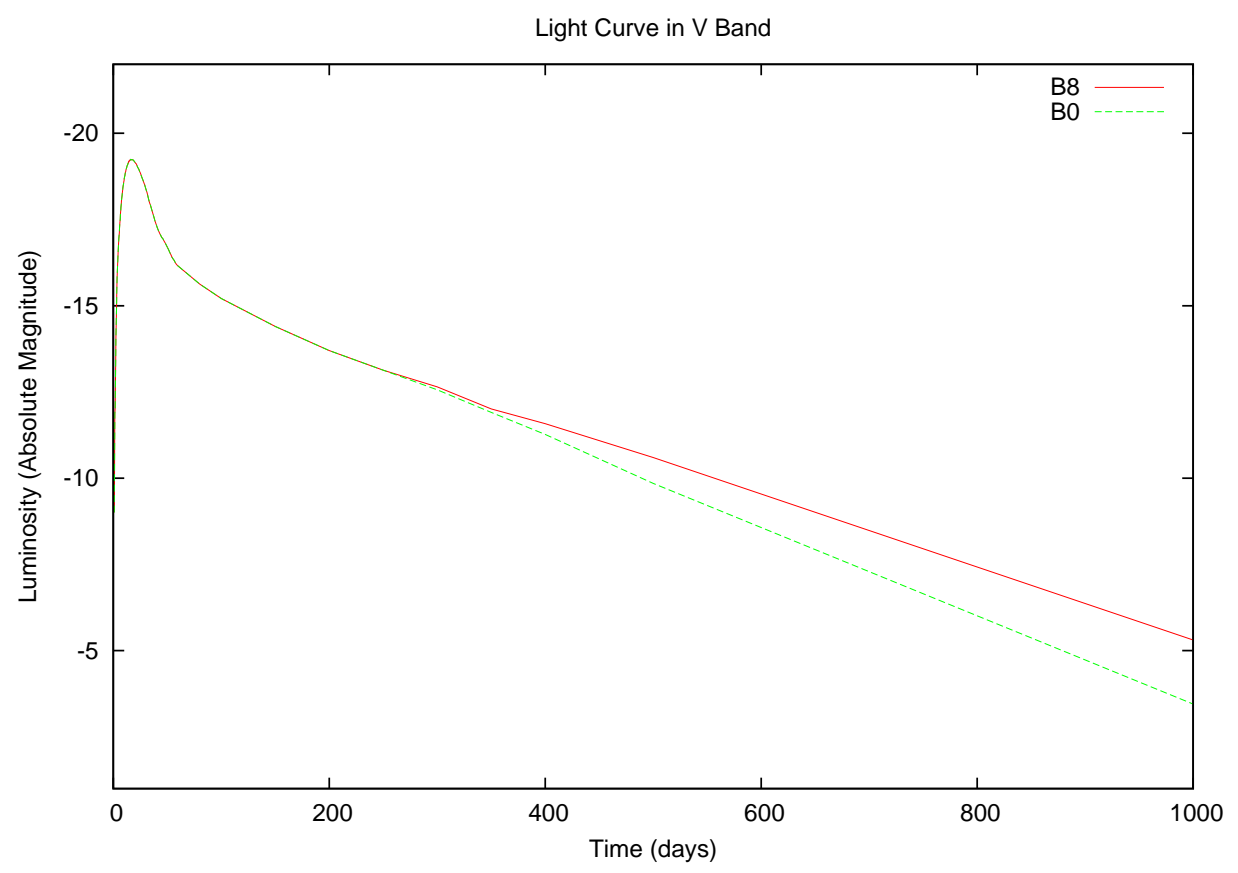

